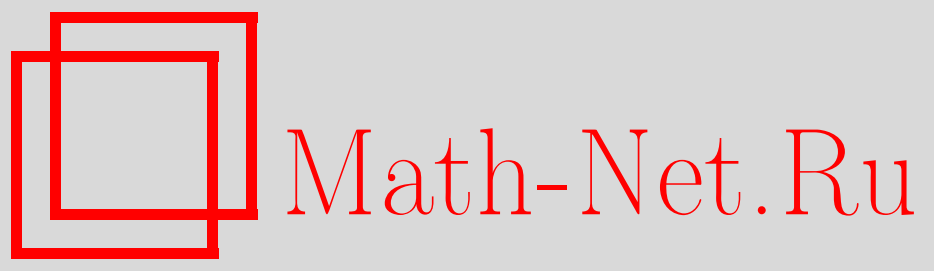

А. Эгбали, А. Резаи-Агдам, Классические $r$-матрицы двух- и трехмерных супербиалгебр Ли и их супергруппы Пуассона-Ли, ТМФ, 2012, том 172, номер 1, 100-121

DOI: https://doi.org/10.4213/tmf6923

Использование Общероссийского математического портала Math-Net.Ru подразумевает, что вы прочитали и согласны с пользовательским соглашением http://www . mathnet.ru/rus/agreement

Параметры загрузки:

IP: 18.209 .158 .208

26 апреля 2023 г., 09:11:30

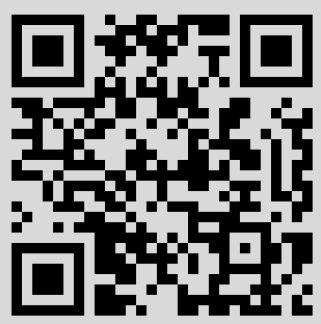




\section{КЛАССИЧЕСКИЕ $r$-МАТРИЦЫ ДВУХ- И ТРЕХМЕРНЫХ СУПЕРБИАЛГЕБР ЛИ И ИХ СУПЕРГРУППЫ ПУАССОНА-ЛИ}

Получены классические $r$-матрицы двух- и трехмерных супербиалгебр Ли. На основании этого классифицируются все двух- и трехмерные кограничные супербиалгебры Ли и их типы (треугольные, квазитреугольные или факторизуемые). С использованием суперскобки Склянина получены суперпуассоновы структуры на связанных с ними супергруппах Пуассона-Ли.

Ключевые слова: супербиалгебра Ли, супергруппа Пуассона-Ли, классическая $r$-матрица.

\section{1. ВВЕДЕНИЕ}

В математике биалгебры Ли впервые были введены Дринфельдом как алгебраические структуры и классический предел квантованных обертывающих алгебр (квантовых груnп) [1]. В частности, каждая деформация универсальной обертывающей алгебры индуцирует структуру биалгебры Ли на соответствующей алгебре Ли. И наоборот, как было показано в работе [2], каждая биалгебра Ли допускает квантование. Поэтому классификацию биалгебр Ли можно рассматривать как первый шаг в классификации квантовых групп. С другой стороны, с физической точки зрения теория классических интегрируемых систем естественным образом связана с геометрией и теорией представлений групп Пуассона-Ли и соответствующими биалгебрами Ли и их классическими $r$-матрицами (см., например, [3]). Более того, биалгебры Ли и группы Пуассона-Ли можно применять в теории $T$-дуальных сигма-моделей Пуассона-Ли [4] и $N=2$ суперконформных теориях поля [5]. Супербиалгебры Ли [6], как алгебры симметрии, играют важную роль в интегрируемых структурах AdS/CFT-соответствия [7], а также в моделях Тоды на супералгебрах Ли [8]. Аналогично можно рассмотреть $T$-дуальные сигма-модели Пуассона-Ли на супергруппах Пуассона-Ли [9]. Таким образом, а также с учетом того, что существует универсальное квантование для супербиалгебр Ли [10], классификация супербиалгебр Ли (особенно супербиалгебр Ли низких размерностей) оказывается важной как с физической, так и с математической точки зрения. В работах [11]

${ }^{*}$ Department of Physics, Faculty of Science, Azarbaijan Shahid Madani University, Tabriz, Iran. E-mail: rezaei-a@azaruniv.edu 
и [12] мы классифицировали все двух- и трехмерные супербиалгебры Ли. В настоящей работе, следуя работам [11] и [12], мы находим классические $r$-матрицы этих супербиалгебр Ли и определяем их типы (треугольные, квазитреугольные или факторизуемые). Более того, мы получаем суперпуассоновы структуры на соответствующих супергруппах Пуассона-Ли.

Работа построена следующим образом. В разделе 2 приведены некоторые основные определения и обозначения для кограничных супербиалгебр Ли и супертроек Манина. В разделе 3 перечислены разложимые и неразложимые супералгебры Ли и связанные с ними супербиалгебры Ли [11], [12]. В разделе 4 с использованием присоединенного и матричного представлений вычисляются классические $r$-матрицы и определяются двух- и трехмерные кограничные (треугольные, квазитреугольные или факторизуемые) супербиалгебры Ли. В разделе 5 мы сначала вычисляем инвариантные супервекторные поля, а затем, используя суперскобки Склянина, получаем суперпуассоновы структуры на двух- и трехмерных супергруппах Пуассона-Ли.

\section{2. ОПРЕДЕЛЕНИЯ И ОБОЗНАЧЕНИЯ}

В настоящей работе мы применяем обозначения де Витта для супервекторных пространств, суперматриц и т. д. [13]. Напомним некоторые основные определения и предложения, касающиеся супербиалгебр Ли [6], [11], [14].

ОПРЕДЕЛЕНИЕ 1. Супералгебра Ли $\mathbf{g}$ является градуированным векторным пространством $\mathbf{g}=\mathbf{g}_{B} \oplus \mathbf{g}_{F}$ с градуировками $\operatorname{grad}\left(\mathbf{g}_{B}\right)=0$ и $\operatorname{grad}\left(\mathbf{g}_{F}\right)=1$, так что скобка Ли удовлетворяет суперантисимметричному тождеству и супертождеству Якоби, т. е. в градуированном базисе $\left\{X_{i}\right\}$ в $\mathbf{g}$ имеем $^{1)}$

$$
\left[X_{i}, X_{j}\right]=f^{k}{ }_{i j} X_{k},
$$

и

$$
(-1)^{i(j+k)} f^{m}{ }_{j l} f_{k i}^{l}+f^{m}{ }_{i l} f_{j k}^{l}+(-1)^{k(i+j)} f_{k l}^{m} f_{i j}^{l}=0,
$$

так что

$$
f^{k}{ }_{i j}=-(-1)^{i j} f^{k}{ }_{j i}
$$

Более того, имеем

$$
f^{k}{ }_{i j}=0, \quad \operatorname{grad}(i)+\operatorname{grad}(j) \neq \operatorname{grad}(k) \quad(\bmod 2) .
$$

Пусть $\mathrm{g}$ - конечномерная супералгебра Ли, $\mathrm{g}^{*}$ - ее дуальное суперпространство относительно невырожденного канонического спаривания $(\cdot, \cdot)$ на $\mathbf{g}^{*} \oplus \mathbf{g}$.

ОПреДЕЛЕНиЕ 2. Структура супербиалгебры Ли на супералгебре Ли g является линейным отображением $\delta: \mathbf{g} \rightarrow \mathbf{g} \otimes \mathbf{g}$ (суперкокоммутатором) таким, что:

1) $\delta$ является супер-1-коциклом, т. е. ${ }^{2)}$

$$
\begin{gathered}
\delta([X, Y])=\left(\operatorname{ad}_{X} \otimes I+I \otimes \operatorname{ad}_{X}\right) \delta(Y)-(-1)^{|X||Y|}\left(\operatorname{ad}_{Y} \otimes I+I \otimes \operatorname{ad}_{Y}\right) \delta(X) \\
\forall X, Y \in \mathbf{g} ;
\end{gathered}
$$

1) Заметим, что скобка в случае пары бозон-бозон или бозон-фермион представляет собой обычный коммутатор, однако в случае пары фермион-фермион она представляет собой антикоммутатор. Более того, мы обозначаем градуировку индексов теми же индексами в степени числа -1 , например $\operatorname{grad}(i) \equiv i$; такие обозначения применял де Витт в работе [13].

2) Здесь $|X|(|Y|)$ обозначает градуировку элемента $X(Y)$. 
2) дуальное отображение ${ }^{t} \delta: \mathbf{g}^{*} \otimes \mathbf{g}^{*} \rightarrow \mathbf{g}^{*}$ является суперскобкой Ли на $\mathbf{g}^{*}$, т. е.

$$
(\xi \otimes \eta, \delta(X))=\left({ }^{t} \delta(\xi \otimes \eta), X\right)=\left([\xi, \eta]_{*}, X\right) \quad \forall X \in \mathbf{g}, \quad \xi, \eta \in \mathbf{g}^{*} .
$$

Супербиалгебру Ли, определеляемую таким способом, будем обозначать как $\left(\mathbf{g}, \mathbf{g}^{*}\right)$ или $(\mathbf{g}, \delta)[6],[11]$.

ОПРЕДЕЛЕНИЕ 3. Супербиалгебра Ли является кограничной, если суперкокоммутатор является 1-кограницей, т. е. если существует элемент $r \in \mathbf{g} \otimes \mathbf{g}$ такой, что

$$
\delta(X)=\left(I \otimes \operatorname{ad}_{X}+\operatorname{ad}_{X} \otimes I\right) r \quad \forall X \in \mathbf{g} .
$$

ПреДЛОЖенИЕ 1. Две кограничные супербиалгебры Ли $\left(\mathbf{g}, \mathbf{g}^{*}\right) u\left(\mathbf{g}^{\prime}, \mathbf{g}^{*}\right)$, определенные с помощъю $r \in \mathbf{g} \otimes \mathbf{g} u r^{\prime} \in \mathbf{g}^{\prime} \otimes \mathbf{g}^{\prime}$, являются изоморфными тогда $и$ только тогда, когда имеется изоморфизм супералгебр Ли $\alpha: \mathbf{g} \rightarrow \mathbf{g}^{\prime}$ такой, что $(\alpha \otimes \alpha) r-r^{\prime}$ есть $\mathbf{g}^{\prime}$-инвариант [14], m.е.

$$
\left(I \otimes \operatorname{ad}_{X}+\operatorname{ad}_{X} \otimes I\right)\left((\alpha \otimes \alpha) r-r^{\prime}\right)=0 \quad \forall X \in \mathbf{g}^{\prime} .
$$

ОПРЕДЕЛЕНиЕ 4. Кограничная супербиалгебра Ли может быть двух различных типов.

А. Если $r$ является суперкососимметричным решением классического уравнения Янга-Бакстера (КУЯБ), т. е. $r^{i j}=-(-1)^{i j} r^{j i}$ и

$$
[[r, r]]=0
$$

то говорят, что кограничная супербиалгебра Ли является треугольной. При этом суперскобка Схоутена определяется как ${ }^{3)}$

$$
[[r, r]]=\left[r_{12}, r_{13}\right]+\left[r_{12}, r_{23}\right]+\left[r_{13}, r_{23}\right],
$$

и если обозначить $r=r^{i j} X_{i} \otimes X_{j}$, то $r_{12}=r^{i j} X_{i} \otimes X_{j} \otimes 1, r_{13}=r^{i j} X_{i} \otimes 1 \otimes X_{j}$ и $r_{23}=r^{i j} 1 \otimes X_{i} \otimes X_{j}$. Решение КУЯБ часто называют классической $r$-матрицей.

Б. Если $r$ - решение КУЯБ такое, что $r_{12}+r_{21}$ является g-инвариантным элементом в $\mathbf{g} \otimes \mathbf{g}$, то говорят, что кограничная супербиалгебра Ли квазитреугольная. Более того, если суперсимметричная часть $r$ обратима, то матрица $r$ называется факторизуемой.

Иногда условие Б можно заменить на следующее.

Б'. Если $r$ - суперкососимметричное решение модифицированного КУЯБ

$$
[[r, r]]=\omega, \quad \omega \in \stackrel{3}{\Lambda}^{g}
$$

то говорят, что кограничная супербиалгебра Ли является квазитреуголъной [6].

Заметим, что если $\mathbf{g}$ - супербиалгебра Ли, то $\mathbf{g}^{*}$ - также супербиалгебра Ли [6], однако это не всегда верно для свойства кограничности.

3) Заметим, что $r$ имеет грассманову четность нуль, а грассманова четность $r^{i j}$ определяется индексами; например, имеем $\left[r_{12}, r_{13}\right]=(-1)^{i(k+l)+j l} r^{i j} r^{k l}\left[X_{i}, X_{k}\right] \otimes X_{j} \otimes X_{l}$. 
ОПРЕДЕЛЕНИЕ 5. Пусть $\mathrm{g}$ - кограничная супербиалгебра Ли с супер-1-коциклом $\delta$, a $\mathbf{g}^{*}$ - также кограничная супербиалгебра Ли с супер-1-коциклом $\delta^{*}$,

$$
\forall \xi \in \mathbf{g}^{*} \quad \exists r^{*} \in \mathbf{g}^{*} \otimes \mathbf{g}^{*}, \quad \delta^{*}(\xi)=\left(I \otimes \operatorname{ad}_{\xi}+\operatorname{ad}_{\xi} \otimes I\right) r^{*},
$$

где $\delta^{*}: \mathbf{g}^{*} \longrightarrow \mathbf{g}^{*} \otimes \mathbf{g}^{*}$. Тогда пара $\left(\mathbf{g}, \mathbf{g}^{*}\right)$ называется би-r-матричной супербиалгеброй Ли [14], если суперскобка Ли $[\cdot, \cdot \cdot]^{\prime}$ на $\mathbf{g}$, определямая с помощью ${ }^{t} \delta^{*}$ как

$$
\left(\delta^{*}(\xi), X \otimes Y\right)=\left(\xi,{ }^{t} \delta^{*}(X \otimes Y)\right)=\left(\xi,[X, Y]^{\prime}\right) \quad \forall X, Y \in \mathbf{g}, \quad \xi \in \mathbf{g}^{*},
$$

эквивалентна исходной [14], т. е.

$$
[X, Y]^{\prime}=S^{-1}[S X, S Y] \quad \forall X, Y \in \mathbf{g}, \quad S \in \operatorname{Aut}(\mathbf{g}) .
$$

ОПРЕДЕЛЕНИЕ 6. Супертройка Манина представляет собой тройку супералгебр Ли $(\mathcal{D}, \mathbf{g}, \tilde{\mathbf{g}})$ вместе с невырожденной аd-инвариантной суперсимметричной билинейной формой $\langle\cdot, \cdot\rangle$ на $\mathcal{D}$ такой, что:

1) $\mathbf{g}$ и $\tilde{\mathbf{g}}$ являются подсупералгебрами Ли супералгебры $\mathcal{D}$;

2) $\mathcal{D}=\mathbf{g} \oplus \tilde{\mathbf{g}}$ как супервекторное пространство;

3) g и $\tilde{\mathbf{g}}$ изотропны относительно $\langle\cdot, \cdot\rangle$, т. е.

$$
\left\langle X_{i}, X_{j}\right\rangle=\left\langle\tilde{X}^{i}, \tilde{X}^{j}\right\rangle=0, \quad \delta_{i}{ }^{j}=\left\langle X_{i}, \tilde{X}^{j}\right\rangle=(-1)^{i j}\left\langle\tilde{X}^{j}, X_{i}\right\rangle=(-1)^{i j} \delta^{j}{ }_{i},
$$

где $\left\{X_{i}\right\}$ и $\left\{\tilde{X}^{i}\right\}$ - базисы супералгебр Ли $\mathbf{g}$ и $\tilde{\mathbf{g}}$ соответственно [6], [11].

Заметим, что в соотношении $(15) \delta^{j}{ }_{i}$ - обыкновенная дельта-функция. Имеется взаимно однозначное соответствие между супербиалгеброй Ли $\left(\mathbf{g}, \mathbf{g}^{*}\right)$ и супертройкой Манина $(\mathcal{D}, \mathbf{g}, \tilde{\mathbf{g}})$, где $\tilde{\mathbf{g}}=\mathbf{g}^{*}$. Если выбрать структурные константы супералгебр Ли $\mathbf{g}$ и $\tilde{\mathbf{g}}$ так, что

$$
\left[X_{i}, X_{j}\right]=f^{k}{ }_{i j} X_{k}, \quad\left[\tilde{X}^{i}, \tilde{X}^{j}\right]=\tilde{f}_{k}^{i j} \tilde{X}^{k},
$$

то из аd-инвариантности билинейной формы $\langle\cdot, \cdot\rangle$ на $\mathcal{D}=\mathbf{g} \oplus \mathbf{g}$ следует, что [11]

$$
\left[X_{i}, \widetilde{X}^{j}\right]=(-1)^{j} \tilde{f}^{j k}{ }_{i} X_{k}+(-1)^{i} f^{j}{ }_{k i} \tilde{X}^{k} .
$$

Очевидно, используя соотношения (6), (15) и (16), имеем

$$
\delta\left(X_{i}\right)=(-1)^{j k} \tilde{f}^{j k}{ }_{i} X_{j} \otimes X_{k} .
$$

Заметим, что множитель $(-1)^{j k}$ появляется в этом соотношении в связи с определением естественного скалярного произведения между $\mathbf{g} \otimes \mathbf{g}$ и $\mathbf{g}^{*} \otimes \mathbf{g}^{*}$ как $\left(\widetilde{X}^{i} \otimes\right.$ $\left.\tilde{X}^{j}, X_{k} \otimes X_{l}\right)=(-1)^{j k} \delta^{i}{ }_{k} \delta^{j}{ }_{l}$. В результате, если мы применим это соотношение супер-1-коцикла к условию (5), то получим супертождества Якоби (2) для дуальной супералгебры Ли и смешанные супертождества Якоби:

$$
f^{m}{ }_{j k} \tilde{f}_{m}^{i l}=f_{m k}^{i} \tilde{f}_{j}^{m l}+f_{j m}^{l} \tilde{f}_{k}^{i m}+(-1)^{j l} f_{j m}^{i} \tilde{f}_{k}^{m l}+(-1)^{i k} f_{m k}^{l} \tilde{f}_{j}^{i m} .
$$

Это соотношение также можно получить из супертождества Якоби для супералгебры $\mathcal{D}$. 


\section{3. ДВУХ- И ТРЕХМЕРНЫЕ СУПЕРАЛГЕБРЫ ЛИ И СУПЕРБИАЛГЕБРЫ ЛИ}

В работе [11] мы получили и классифицировали все двух- и трехмерные супербиалгебры Ли для всех двух- и трехмерных неразложимых супералгебр Ли. Метод классификации является новым и представляет собой усовершенствование и обобщение на супералгебры Ли метода из работы [15] ${ }^{4)}$. В рамках этого метода путем прямого вычисления, используя присоединенное представление супертождества Якоби и смешанных супертождеств Якоби (2) и (19), мы находим дуальные супералгебры Ли; затем, используя лиевские супергруппы автоморфизмов супералгебр Ли, мы классифицируем все неизоморфные двух- и трехмерные супербиалгебры Ли [11], [12]. Для наглядности используемых обозначений и для самосогласованности работы в табл. 1-4 приведены соответственно двух- и трехмерные неразложимые и разложимые супералгебры Ли [17] и связанные с ними супербиалгебры Ли [11], [12] (заметим, что поскольку мы используем в настоящей работе обозначения де Витта и стандартный базис, структурные константы $C_{F F}^{B}$ должны быть чисто мнимыми). В настоящем разделе мы будем использовать классификацию двух- и трехмерных супералгебр Ли, приведенную в работе [17]. В этой классификации супералгебры Ли делятся на два типа: тривиальные и нетривиальные супералгебры Ли, для которых коммутаторы в случае пары фермион-фермион равны или не равны нулю соответственно. Результаты представлены в табл. 1. Как видно из таблицы, $(m, n-m)$ супералгебры Ли имеют $m$ бозонных генераторов $\left\{X_{1}, \ldots, X_{m}\right\}$ и $n-m$ фермионных генераторов $\left\{X_{m+1}, \ldots, X_{n}\right\}$. При нумерации тривиальных супералгебр Ли буквы $A, B$ и $C$ с целочисленными верхними индексами $i$ и вещественными нижними индексами $p$ обозначают классы эквивалентности супералгебр Ли размерности $d$, где $d=1$ для $A, d=2$ для $B$ и $d=3$ для $C$. Верхние индексы $i$ и вещественные нижние индексы $p$ обозначают число неизоморфных супералгебр Ли и параметры супералгебры Ли соответственно. Для нетривиальных супералгебр Ли к символу в скобках для соответствующей тривиальной супералгебры Ли добавляется, где это необходимо, целочисленный верхний индекс и вещественный нижний индекс.

В табл. 1 разложимые супералгебры Ли имеют вид $B+A_{1,1}=B \oplus A_{1,1},\left(2 A_{1,1}+\right.$ $A)=\left(A_{1,1}+A\right) \oplus A_{1,0}, C_{0}^{1}=C_{p=0}^{1}, C_{0}^{2}=C_{p=0}^{2}=B \oplus A_{0,1}$ и $\left(A_{1,1}+2 A\right)^{0}=\left(A_{1,1}+A\right) \oplus$ $A_{0,1}$. Супералгебра Ли $A$ является одномерной абелевой супералгеброй Ли с одним фермионным генератором, причем супералгебра Ли $A_{1,1}$ является ее бозонизацией. Более того, супералгебра $\left(C_{1 / 2}^{1}\right)$ отлична от $C_{p}^{1}$, что мы показываем с помощью $C_{p=1 / 2}^{1}$.

Некоторые из приведенных в табл. 1 супералгебр Ли малых размерностей являются подсупералгебрами Ли классических супералгебр Ли:

$$
\begin{array}{cc}
B \subset g l(1 \mid 1), \operatorname{osp}(1 \mid 2), & \left(A_{1,1}+A\right) \subset \operatorname{osp}(1 \mid 2), \quad B+A_{1,1} \subset g l(1 \mid 1), \\
C_{0}^{1} \subset \operatorname{osp}(1 \mid 2), \quad & C_{p}^{1} \subset \operatorname{osp}(1 \mid 2), \quad\left(C_{1 / 2}^{1}\right) \subset \operatorname{osp}(1 \mid 2), e(2), \\
\left(A_{1,1}+2 A\right)^{0} \subset e(2) .
\end{array}
$$

4) Заметим, что в работе [16], к сожалению, отсутствует стандартный и логичный метод получения биалгебр Ли низких размерностей. 
ТАБлицА 1. Двух- и трехмерные разложимые и неразложимые супералгебры Ли.

\begin{tabular}{|c|c|c|c|c|c|}
\hline Тип & $\mathrm{g}$ & $\begin{array}{l}\text { Бозон- } \\
\text { ный } \\
\text { базис }\end{array}$ & $\begin{array}{l}\text { Фермион- } \\
\text { ный } \\
\text { базис }\end{array}$ & $\begin{array}{c}\text { Ненулевые } \\
(\text { анти)коммутационные } \\
\text { соотношения }\end{array}$ & Комментарии \\
\hline \multirow[t]{2}{*}{$(1,1)$} & $B$ & $X_{1}$ & $X_{2}$ & {$\left[X_{1}, X_{2}\right]=X_{2}$} & TPB \\
\hline & $\left(A_{1,1}+A\right)$ & $X_{1}$ & $X_{2}$ & $\left\{X_{2}, X_{2}\right\}=X_{1}$ & HTP \\
\hline \multirow[t]{7}{*}{$(2,1)$} & $B+A_{1,1}$ & $X_{1}, X_{2}$ & $X_{3}$ & {$\left[X_{1}, X_{3}\right]=X_{3}$} & P3P, TPB \\
\hline & $\left(2 A_{1,1}+A\right)$ & $X_{1}, X_{2}$ & $X_{3}$ & $\left\{X_{3}, X_{3}\right\}=X_{1}$ & НЛП, НТР \\
\hline & $C_{0}^{1}$ & $X_{1}, X_{2}$ & $X_{3}$ & {$\left[X_{1}, X_{2}\right]=X_{2}$} & PЗР, ТРВ \\
\hline & $C_{p}^{1}$ & $X_{1}, X_{2}$ & $X_{3}$ & {$\left[X_{1}, X_{2}\right]=X_{2}$} & $p \neq 0$, ТРВ \\
\hline & $\left(C_{1 / 2}^{1}\right)$ & $X_{1}, X_{2}$ & $X_{3}$ & $\begin{array}{l}{\left[X_{1}, X_{3}\right]=p X_{3}} \\
{\left[X_{1}, X_{2}\right]=X_{2}}\end{array}$ & НTP \\
\hline & $\left(\sigma_{1 / 2}\right)$ & $\Lambda_{1}, \alpha_{2}$ & $\Lambda_{3}$ & {$\left[X_{1}, X_{3}\right]=\frac{1}{2} X_{3}$} & \\
\hline & $C^{2}$ & & & $\left\{X_{3}, X_{3}\right\}=X_{2}$ & \\
\hline \multirow{7}{*}{$(1,2)$} & $\left(A_{1,1}+2 A\right)^{0}$ & $\begin{array}{l}X_{1} \\
X_{1}\end{array}$ & $\begin{array}{l}X_{2}, X_{3} \\
X_{2}, X_{3}\end{array}$ & $\begin{array}{l}{\left[X_{1}, X_{2}\right]=X_{2}} \\
\left\{X_{2}, X_{2}\right\}=X_{1}\end{array}$ & $\begin{array}{l}\text { РЗР, ТРВ } \\
\text { НЛП, НТР }\end{array}$ \\
\hline & $C_{p}^{2}$ & $X_{1}$ & $X_{2}, X_{3}$ & {$\left[X_{1}, X_{2}\right]=X_{2}$} & $0<|p| \leqslant 1, \mathrm{TPB}$ \\
\hline & $C^{3}$ & $X_{1}$ & $X_{2}, X_{3}$ & $\begin{array}{l}{\left[X_{1}, X_{3}\right]=p X_{3}} \\
{\left[X_{1}, X_{3}\right]=X_{2}}\end{array}$ & НЛП, ТРВ \\
\hline & $C^{4}$ & $X_{1}$ & $X_{2}, X_{3}$ & {$\left[X_{1}, X_{2}\right]=X_{2}$} & $\mathrm{TPB}$ \\
\hline & $C_{p}^{5}$ & $X_{1}$ & $X_{2}, X_{3}$ & $\begin{array}{c}{\left[X_{1}, X_{3}\right]=X_{2}+X_{3}} \\
{\left[X_{1}, X_{2}\right]=p X_{2}-X_{3}} \\
{\left[X_{1}, X_{3}\right]=X_{2}+p X_{3}}\end{array}$ & $p \geqslant 0, \mathrm{TPB}$ \\
\hline & $\left(A_{1,1}+2 A\right)^{1}$ & $X_{1}$ & $X_{2}, X_{3}$ & $\left\{X_{2}, X_{2}\right\}=X_{1},\left\{X_{3}, X_{3}\right\}=X_{1}$ & НЛП, НТР \\
\hline & $\left(A_{1,1}+2 A\right)^{2}$ & $X_{1}$ & $X_{2}, X_{3}$ & $\left\{X_{2}, X_{2}\right\}=X_{1},\left\{X_{3}, X_{3}\right\}=-X_{1}$ & НЛП, НТР \\
\hline
\end{tabular}

Примечание: ТРВ - тривиальные, НТР - нетривиальные, РЗР - разрешимые, НЛП нильпотентные.

В табл. 4 все дуальные супералгебры Ли являются неизоморфными. Рассматривая их нижние индексы, эти дуальные супералгебры Ли можно представить посредством следующих обозначений: $\widetilde{\mathcal{G}}_{\alpha \beta \gamma}=\left(A_{1,1}+2 A\right)_{\alpha, \beta, \gamma}^{0},\left(A_{1,1}+2 A\right)_{\alpha, \beta, \gamma}^{1}$ и $\left(A_{1,1}+2 A\right)_{\alpha, \beta, \gamma}^{2}$, где имеют место антикоммутационные соотношения

$$
\left\{\widetilde{X}^{2}, \widetilde{X}^{2}\right\}=\alpha \widetilde{X}^{1}, \quad\left\{\widetilde{X}^{2}, \widetilde{X}^{3}\right\}=\beta \widetilde{X}^{1}, \quad\left\{\widetilde{X}^{3}, \widetilde{X}^{3}\right\}=\gamma \widetilde{X}^{1}
$$

$\alpha, \beta, \gamma=0,1,-1, \epsilon, k, s$.

\section{4. ДВУХ- И ТРЕХМЕРНЫЕ КОГРАНИЧНЫЕ СУПЕРБИАЛГЕБРЫ ЛИ}

В настоящем разделе мы определим, сколько из 74 перечисленных в табл. 2-4 двух- и трехмерных супербиалгебр Ли являются кограничными. Заметим, что здесь мы будем работать в нестандартном базисе, поэтому будем опускать коэффициент $i=\sqrt{-1}$ во всех антикоммутационных соотношениях для супералгебр Ли и супербиалгебр Ли из работ [11] и [12]. Таким образом, мы должны найти $r=r^{i j} X_{i} \otimes X_{j} \in \mathbf{g} \otimes \mathbf{g}$ так, чтобы суперкокоммутатор супербиалгебры Ли можно было записать в виде (7). Используя соотношения (7), (16) и (18), находим

$$
\widetilde{\mathcal{Y}}_{i}=\mathcal{X}_{i}{ }^{\mathrm{st}} r+(-1)^{l} r \mathcal{X}_{i}
$$


ТАБлицА 2. Трехмерные супербиалгебры Ли типа $(2,1)$.

\begin{tabular}{|c|c|c|c|}
\hline $\mathrm{g}$ & $\tilde{\mathrm{g}}$ & $\begin{array}{c}\text { Ненулевые (анти)коммутационные } \\
\text { соотношения в } \tilde{\mathrm{g}} \\
\end{array}$ & Комментарии \\
\hline$\left(2 A_{1,1}+A\right)$ & $I_{(2,1)}$ & & \\
\hline \multirow[t]{4}{*}{$B+A_{1,1}$} & $I_{(2,1)}$ & & \\
\hline & $B+A_{1,1} \mid \cdot i$ & {$\left[\widetilde{X}^{2}, \widetilde{X}^{3}\right]=\widetilde{X}^{3}$} & \\
\hline & $\left(2 A_{1,1}+A\right)$ & $\left\{\widetilde{X}^{3}, \widetilde{X}^{3}\right\}=\widetilde{X}^{1}$ & \\
\hline & $\left(2 A_{1,1}+A\right) \cdot i$ & $\left\{\widetilde{X}^{3}, \widetilde{X}^{3}\right\}=-\widetilde{X}^{1}$ & \\
\hline \multirow[t]{5}{*}{$C_{p}^{1}$} & $I_{(2,1)}$ & & $p \in \mathbb{R}$ \\
\hline & $\left(2 A_{1,1}+A\right)$ & $\left\{\widetilde{X}^{3}, \widetilde{X}^{3}\right\}=\widetilde{X}^{1}$ & $p \in \mathbb{R}$ \\
\hline & $\left(2 A_{1,1}+A\right) \cdot i$ & $\left\{\widetilde{X}^{3}, \widetilde{X}^{3}\right\}=-\widetilde{X}^{1}$ & $p \in \mathbb{R}$ \\
\hline & $\left(2 A_{1,1}+A\right) . i i$ & $\left\{\widetilde{X}^{3}, \widetilde{X}^{3}\right\}=\widetilde{X}^{2}$ & $p=1 / 2$ \\
\hline & $C_{-p}^{1} . i$ & {$\left[\widetilde{X}^{1}, \widetilde{X}^{2}\right]=\widetilde{X}_{\sim}^{1},\left[\widetilde{X}^{2}, \widetilde{X}^{3}\right]=p \widetilde{X}^{3}$} & $p \in \mathbb{R}$ \\
\hline \multirow{7}{*}{$\begin{array}{c}C_{0}^{1} \\
\left(C_{1 / 2}^{1}\right)\end{array}$} & $C_{0, k}^{1}$ & {$\left[\widetilde{X}^{1}, \widetilde{X}^{2}\right]=k \widetilde{X}^{2}$} & $k \in \mathbb{R}-\{0\}$ \\
\hline & $I_{(2,1)}$ & & \\
\hline & $C_{p}^{1} \cdot i_{\left.\right|_{p=-1 / 2}}$ & {$\left[\widetilde{X}^{1}, \widetilde{X}^{2}\right]=\widetilde{X}^{1},\left[\tilde{X}^{2}, \widetilde{X}^{3}\right]=\frac{1}{2} \widetilde{X}^{3}$} & \\
\hline & $\begin{array}{l}C_{p}^{1} \cdot i i_{\left.\right|_{p=-1 / 2}} \\
\quad\left(C_{1 / 2}^{1}\right) \cdot i\end{array}$ & $\begin{array}{c}{\left[\widetilde{X}^{1}, \widetilde{X}^{2}\right]=-\widetilde{X}^{1},\left[\widetilde{X}^{2}, \widetilde{X}^{3}\right]=-\frac{1}{2} \widetilde{X}^{3}} \\
{\left[\widetilde{X}^{1}, \widetilde{X}^{2}\right]=\widetilde{X}^{1},\left[\widetilde{X}^{2}, \widetilde{X}^{3}\right]=-\frac{1}{2} \widetilde{X}^{3}}\end{array}$ & \\
\hline & & $\left\{\widetilde{X}^{3}, \widetilde{X}^{3}\right\}=\widetilde{X}^{1}$ & \\
\hline & $\left(C_{1 / 2}^{1}\right) . i i$ & {$\left[\widetilde{X}^{1}, \widetilde{X}^{2}\right]=-\widetilde{X}^{1},\left[\widetilde{X}^{2}, \widetilde{X}^{3}\right]=\frac{1}{2} \widetilde{X}^{3}$} & \\
\hline & $\left(C_{1 / 2, k}^{1}\right)$ & $\begin{array}{c}{\left[\widetilde{X}^{1}, \widetilde{X}^{2}\right]=k \widetilde{X}^{2},\left[\widetilde{X}^{1}, \widetilde{X}^{3}\right]=\frac{k}{2} \widetilde{X}^{3}} \\
\left\{\widetilde{X}^{3}, \widetilde{X}^{3}\right\}=k \widetilde{X}^{2}\end{array}$ & $k \in \mathbb{R}-\{0\}$ \\
\hline
\end{tabular}

где индекс $l$ соответствует строке матрицы $\mathcal{X}_{i}$, а $\left(\mathcal{X}_{i}\right)_{l}{ }^{k}=-f_{i l}{ }^{k}$ и $\left(\widetilde{\mathcal{Y}}_{i}\right)^{j k}=-\tilde{f}^{j k}{ }_{i}$ являются присоединенными представлениями для супералгебр Ли $\mathbf{g}$ и $\mathbf{g}$ соответственно (здесь верхний индекс "st" обозначает супертранспонирование). Теперь, используя приведенные выше соотношения, можно найти $r$-матрицы супербиалгебр Ли. Таким образом, мы можем определить, какие из представленных в табл. 2-4 супербиалгебр Ли являются кограничными, и получить их $r$-матрицы. Мы также проделаем это

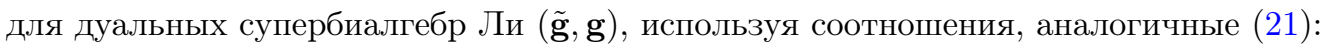

$$
\mathcal{Y}^{i}=\left(\tilde{\mathcal{X}}^{i}\right)^{\mathrm{st}} \tilde{r}+(-1)^{l} \tilde{r} \tilde{\mathcal{X}}^{i},
$$

где, как и ранее, $\left(\tilde{\mathcal{X}}^{i}\right)^{j}{ }_{l}=-\tilde{f}^{i j}{ }_{l}$ и $\left(\mathcal{Y}^{i}\right)_{j k}=-f_{j k}{ }^{i}$. Результаты собраны в табл. 5-7. Заметим, что для определения суперскобок Схоутена на супергруппах Пуассона-Ли

ТАБлицА 3. Двумерные супербиалгебры Ли типа $(1,1)$.

\begin{tabular}{|c|c|c|}
\hline $\mathbf{g}$ & $\tilde{\mathbf{g}}$ & (Анти)коммутационные соотношения в $\tilde{\mathbf{g}}$ \\
\hline$\left(A_{1,1}+A\right)$ & $I_{(1,1)}$ & \\
$B$ & $I_{(1,1)}$ & $\left\{\widetilde{X}^{2}, \widetilde{X}^{2}\right\}=\widetilde{X}^{1}$ \\
& $\left(A_{1,1}+A\right)$ & $\left\{\widetilde{X}^{2}, \widetilde{X}^{2}\right\}=-\widetilde{X}^{1}$ \\
\hline
\end{tabular}


ТАБлица 4. Трехмерные супербиалгебры Ли типа $(1,2)$, где $\epsilon= \pm 1$.

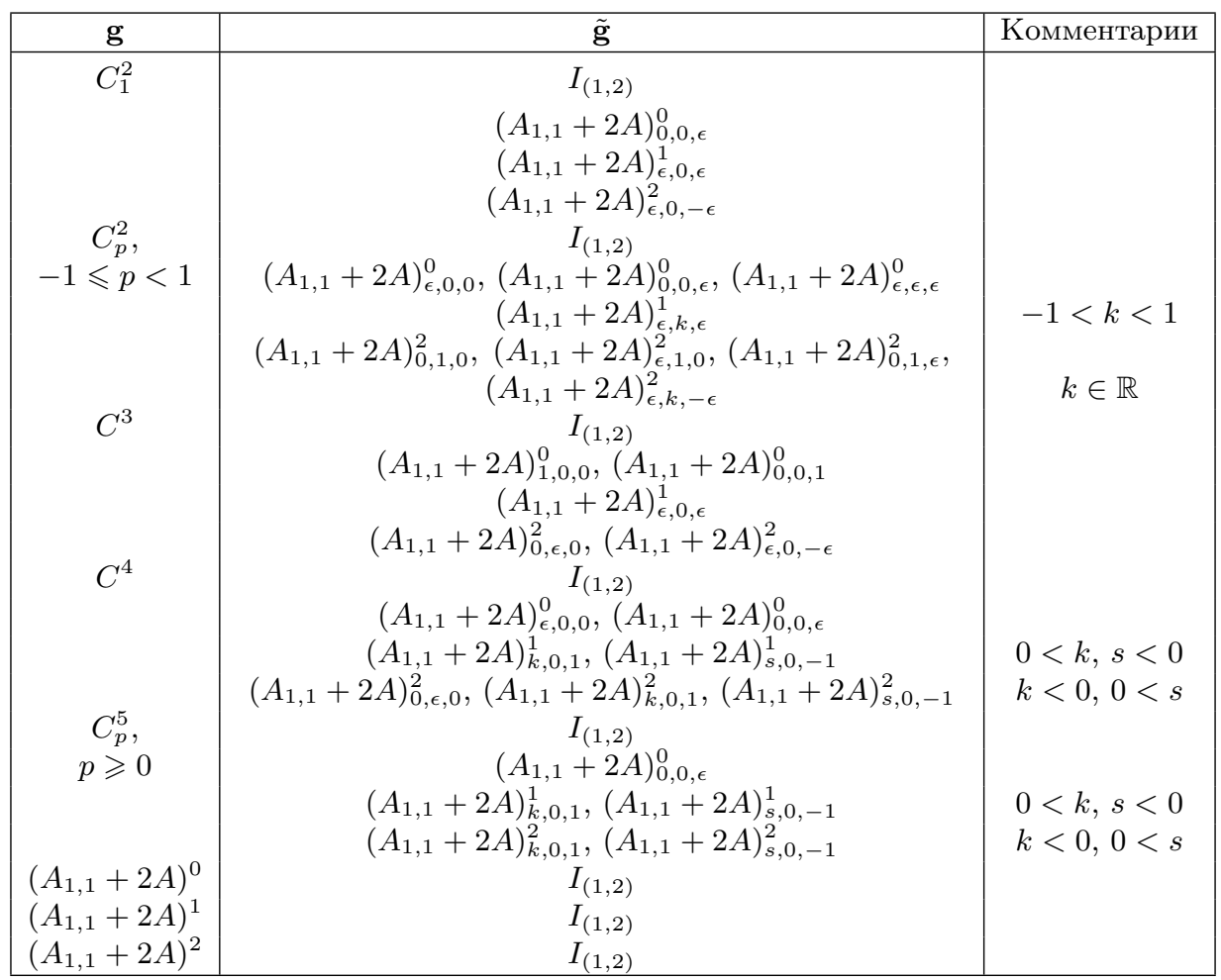

важна информация о типе супербиалгебры Ли (является ли она треугольной или квазитреугольной). В результате мы классифицируем все типы двух- и трехмерных кограничных супербиалгебр Ли. Следует подчеркнуть два момента. Во-первых, во всех таблицах мы выписываем все кограничные супербиалгебры Ли (g, g) с кограничными дуальными $(\tilde{\mathbf{g}}, \mathbf{g})$. Поскольку такие структуры можно задать (с точностью до автоморфизма) с помощью пар $r$-матриц, естественно называть их би- $r$-матричными супербиалгебрами [14]. Здесь мы приводим полный список двух- и трехмерных кограничных и би- $r$-матричных супербиалгебр. Во-вторых, очевидно, что в табл. 5-7 мы рассмотрели суперкососимметричные $r$-матричные решения. Конечно, имеются другие решения для некоторых супербиалгебр Ли из этих таблиц. Эти решения также приведены в таблицах.

Как показано в табл. 5, имеются две би- $r$-матричные супербиалгебры Ли такие, что супербиалгебры Ли $\left(B,\left(A_{1,1}+A\right)\right)$ и $\left(B,\left(A_{1,1}+A\right) . i\right)$ являются треугольными, тогда как дуальные к ним, т. е. супербиалгебры Ли $\left(\left(A_{1,1}+A\right), B\right)$ и $\left(\left(A_{1,1}+A\right) . i, B\right)$, являются квазитреугольными. Более того, чтобы получить суперкососимметричные суперскобоки Пуассона для $\left(\left(A_{1,1}+A\right), I_{(1,1)}\right)$, мы должны положить $a=0$, т. е. $r=0$, в этом случае мы имеем тривиальное решение.

В табл. $6\left(C_{1 / 2, \epsilon=1}^{1}\right)$ и $\left(C_{1 / 2, \epsilon=-1}^{1}\right)$ обозначают дуальные супералгебры Ли для $\left(C_{1 / 2}^{1} . i\right)$ и $\left(C_{1 / 2}^{1} . i i\right)$ соответственно; $\left(\left(2 A_{1,1}+A\right), I_{(2,1)}\right)$ - треугольные супербиалгебры Ли такие, что, как показано в разделе 5 , для получения суперкососимметричных 
ТАБлицА 5. Двумерные кограничные и би- $r$-матричные супербиалгебры Ли типа $(1,1)$.

\begin{tabular}{|c|c|c|c|c|c|}
\hline$(\mathbf{g}, \tilde{\mathbf{g}})$ & $r$ & {$[[r, r]]$} & $\tilde{r}$ & {$[[\tilde{r}, \tilde{r}]]$} & $\begin{array}{c}\text { Коммен- } \\
\text { тарии }\end{array}$ \\
\hline$\left(\left(A_{1,1}+A\right), I_{(1,1)}\right)$ & $a X_{1} \otimes X_{1}$ & 0 & & & $a \in \mathbb{R}$ \\
$\left(B,\left(A_{1,1}+A\right)\right)$ & $-\frac{1}{4} X_{2} \wedge X_{2}$ & 0 & $a \widetilde{X}^{1} \otimes \widetilde{X}^{1}+\frac{1}{2} \widetilde{X}^{2} \wedge \widetilde{X}^{2}$ & $-\frac{1}{2} \widetilde{X}^{1} \wedge \widetilde{X}^{2} \wedge \widetilde{X}^{2}$ & $a \in \mathbb{R}$ \\
$\left(B,\left(A_{1,1}+A\right) \cdot i\right)$ & $\frac{1}{4} X_{2} \wedge X_{2}$ & 0 & $a \widetilde{X}^{1} \otimes \widetilde{X}^{1}-\frac{1}{2} \widetilde{X}^{2} \wedge \widetilde{X}^{2}$ & $\frac{1}{2} \widetilde{X}^{1} \wedge \widetilde{X}^{2} \wedge \widetilde{X}^{2}$ & $a \in \mathbb{R}$ \\
\hline
\end{tabular}

суперскобок Пуассона следует положить $a=d=0, b=-c$, так что ее $r$-матрица будет иметь вид $r=b X_{1} \wedge X_{2}$.

Заметим, что, воообще говоря, мы имеем

$X_{i} \wedge X_{j} \wedge X_{k}=X_{i} \otimes X_{j} \otimes X_{k}+(-1)^{i(j+k)} X_{j} \otimes X_{k} \otimes X_{i}+(-1)^{k(i+j)} X_{k} \otimes X_{i} \otimes X_{j}-$ $-(-1)^{j k} X_{i} \otimes X_{k} \otimes X_{j}-(-1)^{i j} X_{j} \otimes X_{i} \otimes X_{k}-(-1)^{i j+i k+j k} X_{k} \otimes X_{j} \otimes X_{i}$.

ТАБлицА 6. Трехмерные кограничные и би- $r$-матричные супербиалгебры Ли типа $(2,1)$, где $\zeta, \eta$ являются $a$-числами.

\begin{tabular}{|c|c|c|}
\hline$(\mathrm{g}, \tilde{\mathrm{g}})$ & $r$ & Комментарии \\
\hline$\left(\left(2 A_{1,1}+A\right), I_{(2,1)}\right)$ & $\begin{array}{c}r=a X_{1} \otimes X_{1}+b X_{1} \otimes X_{2}+c X_{2} \otimes X_{1}+d X_{2} \otimes X_{2} \\
{[[r, r]]=0}\end{array}$ & $a, b, c, d \in \mathbb{R}$ \\
\hline$\left(B+A_{1,1}, I_{(2,1)}\right)$ & $\begin{array}{c}r=a X_{2} \otimes X_{2} \\
{[[r, r]]=0}\end{array}$ & $a \in \mathbb{R}$ \\
\hline$\left(B+A_{1,1}, B+A_{1,1} \mid . i\right)$ & $\begin{array}{c}r=a X_{2} \otimes X_{2}+X_{1} \wedge X_{2} \\
{[[r, r]]=0}\end{array}$ & $a \in \mathbb{R}$ \\
\hline & $\begin{array}{c}\tilde{r}=b \widetilde{X}^{1} \otimes \tilde{X}^{1}-\widetilde{X}^{1} \wedge \tilde{X}^{2} \\
{[[\tilde{r}, \tilde{r}]]=0}\end{array}$ & $b \in \mathbb{R}$ \\
\hline$\left(B+A_{1,1},\left(2 A_{1,1}+A\right)\right)$ & $\begin{array}{c}r=a X_{2} \otimes X_{2}-\frac{1}{4} X_{3} \wedge X_{3} \\
{[[r, r]]=0}\end{array}$ & $a \in \mathbb{R}$ \\
\hline & $\begin{array}{c}\tilde{r}=b \widetilde{X}^{1} \otimes \widetilde{X}^{1}+c \widetilde{X}^{1} \otimes \widetilde{X}^{2}+ \\
+d \widetilde{X}^{2} \otimes \widetilde{X}^{1}+e \widetilde{X}^{2} \otimes \widetilde{X}^{2}+\frac{1}{2} \widetilde{X}^{3} \wedge \widetilde{X}^{3} \\
{[[\tilde{r}, \tilde{r}]]=-\frac{1}{2} \widetilde{X}^{1} \wedge \widetilde{X}^{3} \wedge \widetilde{X}^{3}}\end{array}$ & $b, c, d, e \in \mathbb{R}$ \\
\hline$\left(B+A_{1,1},\left(2 A_{1,1}+A\right) \cdot i\right)$ & $\begin{array}{c}r=a X_{2} \otimes X_{2}+\frac{1}{4} X_{3} \wedge X_{3} \\
{[[r, r]]=0}\end{array}$ & $a \in \mathbb{R}$ \\
\hline & $\begin{array}{c}\tilde{r}=b \widetilde{X}^{1} \otimes \widetilde{X}^{1}+c \widetilde{X}^{1} \otimes \widetilde{X}^{2}+ \\
+d \widetilde{X}^{2} \otimes \widetilde{X}^{1}+e \widetilde{X}^{2} \otimes \widetilde{X}^{2}-\frac{1}{2} \widetilde{X}^{3} \wedge \widetilde{X}^{3} \\
\quad[[\tilde{r}, \tilde{r}]]=\frac{1}{2} \widetilde{X}^{1} \wedge \widetilde{X}^{3} \wedge \widetilde{X}^{3}\end{array}$ & $b, c, d, e \in \mathbb{R}$ \\
\hline$\left(C_{p}^{1},\left(2 A_{1,1}+A\right)\right)$ & $\begin{array}{c}r_{1}=-\frac{1}{4 p} X_{3} \wedge X_{3} \\
{\left[\left[r_{1}, r_{1}\right]\right]=0}\end{array}$ & $p \in \mathbb{R}-\{0,1\}$ \\
\hline & $\begin{array}{c}r_{2}=\zeta X_{2} \otimes X_{3}+\eta X_{3} \otimes X_{2}-\frac{1}{4} X_{3} \wedge X_{3} \\
{\left[\left[r_{2}, r_{2}\right]\right]=0}\end{array}$ & $p=1$ \\
\hline
\end{tabular}


Продолжение табл. 6

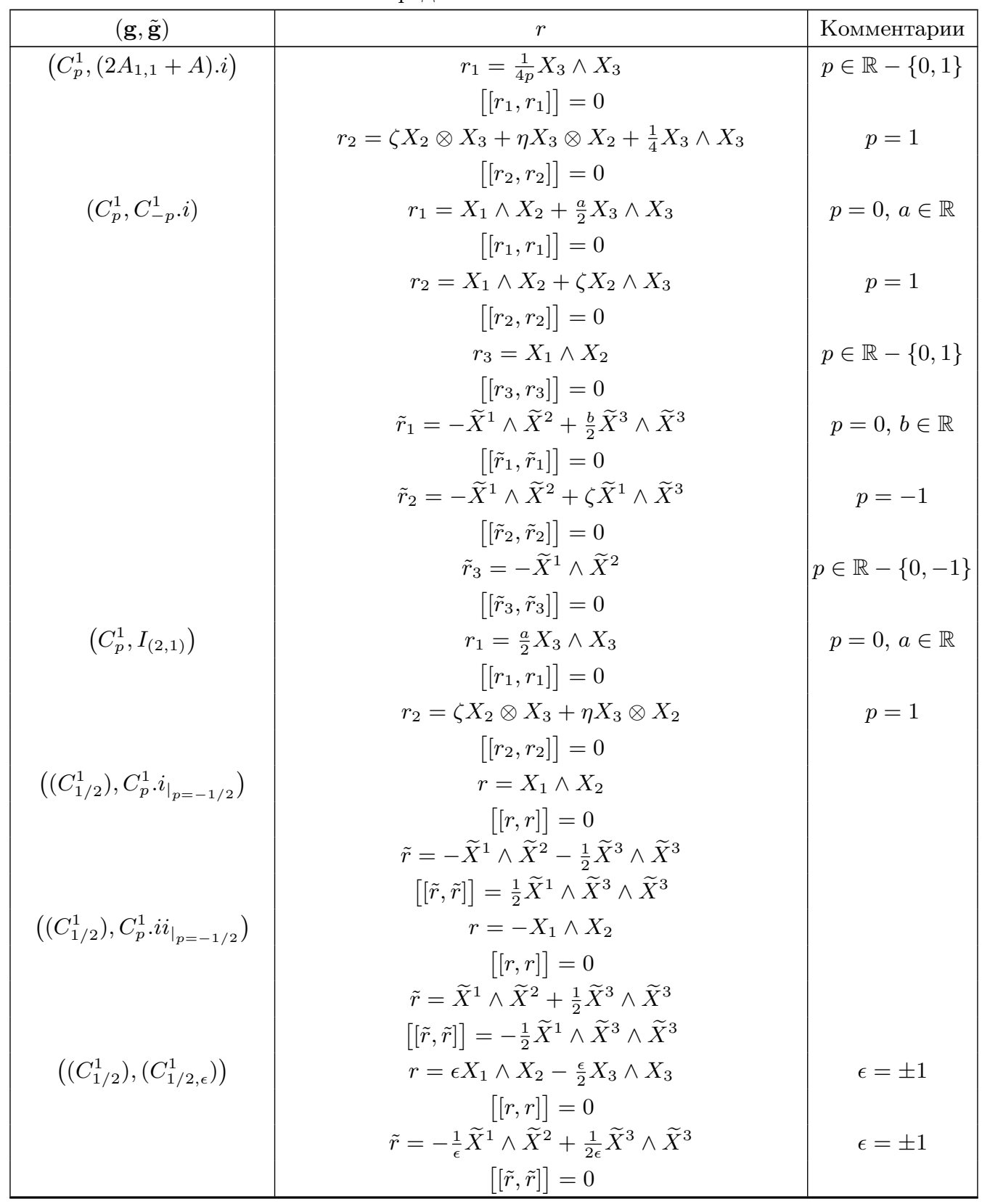

Аналогично для треугольной супербиалгебры Ли $\left(B+A_{1,1}, I_{(2,1)}\right)$ имеем $r=0$, для би- $r$-матричной супербиалгебры Ли $\left(B+A_{1,1}, B+A_{1,1} \mid . i\right)$ треугольного типа имеем $r=X_{1} \wedge X_{2}$ (т. е. $\left.a=0\right)$ и $\tilde{r}=-\widetilde{X}^{1} \wedge \widetilde{X}^{2}$ (т. е. $\left.b=0\right)$. В случае би- $r$-матричных супербиалгебр Ли $\left(B+A_{1,1},\left(2 A_{1,1}+A\right)\right)$ и $\left(B+A_{1,1},\left(2 A_{1,1}+A\right) . i\right)$ треугольного типа и квазитреугольного типа для дуальных к ним имеем $r=-\frac{1}{4} X_{3} \wedge X_{3}$ (т. е. $\left.a=0\right)$ и $r=\frac{1}{4} X_{3} \wedge X_{3}$ (т. е. $\left.a=0\right)$ соответственно. 
ТАБлицА 7. Трехмерные кограничные и би- $r$-матричные супербиалгебры Ли типа $(1,2)$, где $\zeta, \eta$ являются $a$-числами.

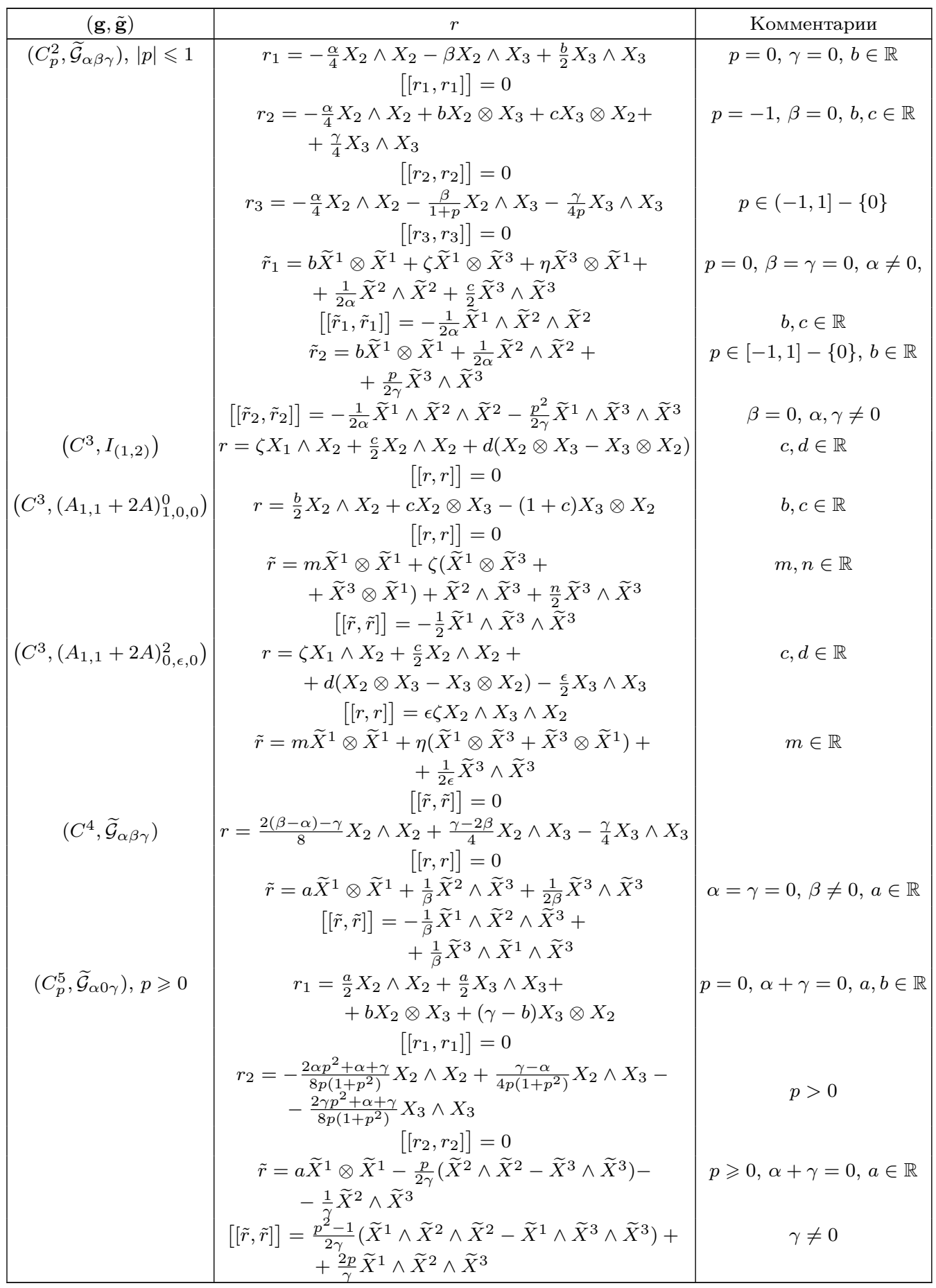


Продолжение табл. 7

\begin{tabular}{|c|c|c|}
\hline$(\mathbf{g}, \tilde{\mathbf{g}})$ & $r$ & Комментарии \\
\hline$\left(\left(A_{1,1}+2 A\right)^{0}, I_{(1,2)}\right)$ & $r=a X_{1} \otimes X_{1}+\zeta X_{1} \otimes X_{3}+\eta X_{3} \otimes X_{1}+$ & $a, d \in \mathbb{R}$ \\
& $+\frac{d}{2} X_{3} \wedge X_{3}$ & \\
$\left(\left(A_{1,1}+2 A\right)^{1}, I_{(1,2)}\right)$ & {$[[r, r]]=0$} & $a \in \mathbb{R}$ \\
$\left(\left(A_{1,1}+2 A\right)^{2}, I_{(1,2)}\right)$ & $r=a X_{1} \otimes X_{1}$ & \\
& $r=a X_{1} \otimes X_{1}+\zeta\left[X_{1} \otimes X_{2}+\right.$ & $a \in \mathbb{R}$ \\
& $\left.+X_{2} \otimes X_{1} \pm X_{1} \otimes X_{3} \pm X_{3} \otimes X_{1}\right]$ & \\
& {$[[r, r]]=0$} & \\
\hline
\end{tabular}

Заметим, что супербиалгебры Ли $\left(C_{p}^{1},\left(2 A_{1,1}+A\right)\right)$ и $\left(C_{p}^{1},\left(2 A_{1,1}+A\right) . i\right)$ являются треугольными и не имеют кограничных дуальных. Более того, чтобы получить суперкососимметричные суперскобки Пуассона, мы должны положить $r_{2}=\zeta X_{2} \wedge$ $X_{3} \mp \frac{1}{4} X_{3} \wedge X_{3}$ (при $p=1$ ), т. е. $\eta=-\zeta$. Супербиалгебры Ли $\left(C_{p}^{1}, C_{-p}^{1} . i\right)$ и дуальные к ним при значении $p=0\left(r_{1}\right.$ и $\left.\tilde{r}_{1}\right), p=1$ ( $r_{2}$ и $\left.\tilde{r}_{3_{\mid p=1}}\right), p=-1\left(r_{3_{\mid p=-1}}\right.$ и $\left.\tilde{r}_{2}\right)$ и $p \in \mathbb{R}-$ $\{-1,0,1\}\left(r_{3}\right.$ и $\left.\tilde{r}_{3}\right)$ являются би- $r$-матричными супербиалгебрами Ли треугольного типа.

Супербиалгебры $\left(C_{p}^{1}, I_{(2,1)}\right)$ являются треугольными при $p=0$ и $p=1$; более того, чтобы получить суперкососимметричные суперскобки Пуассона, мы должны положить $r_{2}=\zeta X_{2} \wedge X_{3}$, т. е. $\eta=-\zeta$. Далее, $\left(\left(C_{1 / 2}^{1}\right), C_{p}^{1} \cdot i_{\left.\right|_{p=-1 / 2}}\right)$ и $\left(\left(C_{1 / 2}^{1}\right), C_{p}^{1} . i i_{\left.\right|_{p=-1 / 2}}\right)$ являются би- $r$-матричными супербиалгебрами Ли треугольного типа, в то время как дуальные к ним являются квазитреугольными. Супербиалгебры Ли $\left(\left(C_{1 / 2}^{1}\right),\left(C_{1 / 2, \epsilon}^{1}\right)\right)$ и дуальные к ним являются би- $r$-матричными супербиалгебрами Ли треугольного типа.

Трехмерные супербиалгебры Ли вида $\left(\mathbf{g}, \widetilde{\mathcal{G}}_{\alpha \beta \gamma}\right)$, где $\mathbf{g}=C_{p}^{2}, C^{3}, C^{4}, C_{p}^{5}$ и $\widetilde{\mathcal{G}}_{\alpha \beta \gamma}-$ одна из супербиалгебр, дуальных к супералгебрам Ли, из табл. 4 , приведены в табл. 7. Мы видим, что при $p=0$ супербиалгебры Ли $\left(C_{p}^{2}, \widetilde{\mathcal{G}}_{\alpha \beta \gamma}\right)$ являются би- $r$-матричными только при $\beta=\gamma=0$ и $\alpha \neq 0$ (т. е. при $\widetilde{\mathcal{G}}_{\alpha \beta \gamma}=\left(A_{1,1}+2 A\right)_{\epsilon, 0,0}^{0}$ из табл. 4). Заметим, что супербиалгебры $\left(C_{p=0}^{2},\left(A_{1,1}+2 A\right)_{\epsilon, 0,0}^{0}\right)$ являются треугольными, тогда как дуальные к ним - квазитреугольными. Более того, супербиалгебры Ли $\left(C_{p=0}^{2}, \widetilde{\mathcal{G}}_{\alpha, \beta, 0}\right)$ (т. е. $\left.\widetilde{\mathcal{G}}_{\alpha, \beta, 0}=I_{(1,2)},\left(A_{1,1}+2 A\right)_{0,1,0}^{2},\left(A_{1,1}+2 A\right)_{\epsilon, 1,0}^{2}\right)$ являются треугольными. При $p=-1$ супербиалгебры Ли $\left(C_{p}^{2}, \widetilde{\mathcal{G}}_{\alpha \beta \gamma}\right)$ при $\beta=0$ и $\alpha, \gamma \neq 0$ (т. е. $\left.\widetilde{\mathcal{G}}_{\alpha \neq 0, \beta=0, \gamma \neq 0}=\left(A_{1,1}+2 A\right)_{\epsilon, 0, \epsilon}^{1},\left(A_{1,1}+2 A\right)_{\epsilon, 0,-\epsilon}^{2}\right)$ являются би- $r$-матричными, причем $\left(C_{p=-1}^{2},\left(A_{1,1}+2 A\right)_{\epsilon, 0, \epsilon}^{1}\right)$ и $\left(C_{p=-1}^{2},\left(A_{1,1}+2 A\right)_{\epsilon, 0,-\epsilon}^{2}\right)$ являются треугольными, тогда как дуальные к ним - квазитреугольными. Заметим, что при $\beta=\gamma=0$ супербиалгебры Ли $\left(C_{p=-1}^{2},\left(A_{1,1}+2 A\right)_{\epsilon, 0,0}^{0}\right)$ являются треугольными $\left(r_{2}\right)$. Более того, чтобы получить суперкососимметричные суперскобки Пуассона, следует положить $b=c$ в $r_{2}$. Для $p \in(-1,1]-\{0\}$ при $\beta=0$ и $\alpha, \gamma \neq 0$ супербиалгебры Ли $\left(C_{p}^{2},\left(A_{1,1}+2 A\right)_{\epsilon, 0, \epsilon}^{1}\right)$ и $\left(C_{p}^{2},\left(A_{1,1}+2 A\right)_{\epsilon, 0,-\epsilon}^{2}\right)$ являются би- $r$-матричными, имеющими треугольный тип $\left(r_{3}\right)$, в то время как дуальные к ним - квазитреугольного $\left(\tilde{r}_{2}\right)$. С другой стороны, супербиалгебры Ли $\left(C_{p}^{2}, I_{(1,2)}\right),\left(C_{p}^{2},\left(A_{1,1}+2 A\right)_{\epsilon, 0,0}^{0}\right)$, $\left(C_{p}^{2},\left(A_{1,1}+2 A\right)_{0,0, \epsilon}^{0}\right),\left(C_{p}^{2},\left(A_{1,1}+2 A\right)_{\epsilon, \epsilon, \epsilon}^{0}\right),\left(C_{p}^{2},\left(A_{1,1}+2 A\right)_{\epsilon, k \neq 0, \epsilon}^{1}\right),\left(C_{p}^{2},\left(A_{1,1}+2 A\right)_{0,1,0}^{2}\right)$, $\left(C_{p}^{2},\left(A_{1,1}+2 A\right)_{\epsilon, 1,0}^{2}\right),\left(C_{p}^{2},\left(A_{1,1}+2 A\right)_{0,1, \epsilon}^{2}\right)$ и $\left(C_{p}^{2},\left(A_{1,1}+2 A\right)_{\epsilon, k \neq 0,-\epsilon}^{2}\right)$ являются треугольными. 
Заметим, что $\left(C^{3}, I_{(1,2)}\right)$ являются треугольными супербиалгебрами Ли, тогда как $\left(C^{3},\left(A_{1,1}+2 A\right)_{1,0,0}^{0}\right)$ и $\left(C^{3},\left(A_{1,1}+2 A\right)_{0, \epsilon, 0}^{2}\right)$ являются треугольными би- $r$-матричными супербиалгебрами Ли, а дуальные к ним - квазитреугольными. Более того, чтобы получить суперкососимметричные суперскобки Пуассона для $\left(C^{3},\left(A_{1,1}+2 A\right)_{1,0,0}^{0}\right)$, следует положить $c=-1 / 2$ в $r$, а для $\left(C^{3},\left(A_{1,1}+2 A\right)_{0, \epsilon, 0}^{2}\right)$ следует положить $m=\eta=0$ в $\tilde{r}$.

Супербиалгебры Ли $\left(C^{4}, \widetilde{\mathcal{G}}_{\alpha \beta \gamma}\right)$ при $\alpha=\gamma=0$ и $\beta \neq 0$ (т. е. $\widetilde{\mathcal{G}}_{\alpha=0, \beta, \gamma=0}=\left(A_{1,1}+\right.$ $\left.2 A)_{0, \epsilon, 0}^{2}\right)$ являются би- $r$-матричными треугольного типа, в то время как дуальные к ним являются квазитреугольными. При $\beta=0$ имеем треугольные супербиалгебры Ли $\left(C^{4}, \widetilde{\mathcal{G}}_{\alpha \beta \gamma}\right)$ (т. е. $\widetilde{\mathcal{G}}_{\alpha, \beta, \gamma}=I_{(1,2)},\left(A_{1,1}+2 A\right)_{\epsilon, 0,0}^{0},\left(A_{1,1}+2 A\right)_{0,0, \epsilon}^{0},\left(A_{1,1}+2 A\right)_{k, 0,1}^{1}$, $\left.\left(A_{1,1}+2 A\right)_{s, 0,-1}^{1},\left(A_{1,1}+2 A\right)_{k, 0,1}^{2},\left(A_{1,1}+2 A\right)_{s, 0,-1}^{2}\right)$.

Супербиалгебры Ли $\left(C_{p}^{5}, \widetilde{\mathcal{G}}_{\alpha, \beta=0, \gamma}\right)$ при $p=0, \alpha+\gamma=0$ и $\gamma \neq 0$ (т. е. $\widetilde{\mathcal{G}}_{\alpha, \beta=0, \gamma}=$ $\left.\left(A_{1,1}+2 A\right)_{k, 0,1}^{2},\left(A_{1,1}+2 A\right)_{s, 0,-1}^{2}\right)$ являются би- $r$-матричными $\left(r_{1}, \tilde{r}\right)$ треугольного типа, в то время как дуальные к ним являются квазитреугольными. Более того, чтобы получить суперкососимметричные суперскобки Пуассона, мы должны положить $b=\gamma / 2$ в $r_{1}$. Заметим, что при $p>0, \alpha+\gamma=0$ и $\gamma \neq 0$ супербиалгебры Ли $\left(C_{p}^{5},\left(A_{1,1}+2 A\right)_{k, 0,1}^{2}\right),\left(C_{p}^{5},\left(A_{1,1}+2 A\right)_{s, 0,-1}^{2}\right)$ являются би- $r$-матричными $\left(r_{2}, \tilde{r}\right)$ треугольного типа, тогда как дуальные к ним являются квазитреугольными. В то же время при $p>0$ и $\alpha, \beta, \gamma=0,1,-1, \epsilon, k, s$, т. е. супералгебры $\widetilde{\mathcal{G}}_{\alpha \beta \gamma}=I_{(1,2)}$, $\left(A_{1,1}+2 A\right)_{0,0, \epsilon}^{0},\left(A_{1,1}+2 A\right)_{k, 0,1}^{1},\left(A_{1,1}+2 A\right)_{s, 0,-1}^{1},\left(A_{1,1}+2 A\right)_{k, 0,1}^{2},\left(A_{1,1}+2 A\right)_{s, 0,-1}^{2}$ являются треугольными.

Наконец, супербиалгебры Ли $\left(\left(A_{1,1}+2 A\right)^{0}, I_{(1,2)}\right),\left(\left(A_{1,1}+2 A\right)^{1}, I_{(1,2)}\right)$ и $\left(\left(A_{1,1}+\right.\right.$ $\left.2 A)^{2}, I_{(1,2)}\right)$ являются треугольными. Для того чтобы получить суперкососимметричные суперскобки Пуассона, следует положить $a=0, \eta=-\zeta$ для супербиалгебры Ли $\left(\left(A_{1,1}+2 A\right)^{0}, I_{(1,2)}\right)$, т. е. $r=\zeta X_{1} \wedge X_{3}+\frac{d}{2} X_{3} \wedge X_{3}$, для $\left(\left(A_{1,1}+2 A\right)^{1}, I_{(1,2)}\right)-$ $a=0$ (т. е. $r=0)$, а для $\left(\left(A_{1,1}+2 A\right)^{2}, I_{(1,2)}\right)-a=b=0$ (т. е. $\left.r=0\right)$; здесь в двух последних случаях мы имеем тривиальные решения.

Все эти кограничные супербиалгебры Ли являются неизоморфными. В предыдущем разделе мы упоминали условия (8), при которых кограничные супербиалгебры Ли являются изоморфными. Здесь мы рассмотрим эти условия более точно и неформально. Используя матричный вид изоморфизма $\alpha: \mathbf{g} \rightarrow \mathbf{g}^{\prime}$

$$
\alpha\left(X_{i}\right)=(-1)^{j} \alpha_{i}{ }^{j} X^{\prime}{ }_{j}
$$

соотношение (8) можно переписать как

$$
\begin{array}{r}
{\left[\mathcal{X}_{i}^{\prime \text { st }}\left((-1)^{l+k+k j} \alpha^{\mathrm{st}} r^{\mathrm{st}} \alpha-(-1)^{k+l k} r^{\prime \mathrm{st}}\right)\right]^{\mathrm{st}}=} \\
\quad=-\mathcal{X}_{i}^{\prime \mathrm{st}}\left[(-1)^{n+j+j k} \alpha^{\mathrm{st}} r \alpha-(-1)^{j+j n} r^{\prime}\right]
\end{array}
$$

где в левой части индексы $k$ и $j$ обозначают строку и столбец матрицы $r^{\text {st }}, l$ - столбец матрицы $\alpha$, а в правой части индексы $j$ и $k$ обозначают строку и столбец матрицы $r$, $n$ - столбец матрицы $\alpha$. Если приведенные выше матричные соотношения выполнены, то две кограничные супербиалгебры Ли $(\mathbf{g}, \tilde{\mathbf{g}})$ и $\left(\mathbf{g}^{\prime}, \tilde{\mathbf{g}}^{\prime}\right)$ являются изоморфными. Таким образом, можно исследовать эти соотношения для всех кограничных супербиалгебр Ли из таблиц и увидеть, что все они являются неизоморфными. 


\section{5. ВЫЧИСЛЕНИЕ СУПЕРПУАССОНОВЫХ СТРУКТУР С ПОМОЩЬЮ СУПЕРСКОБКИ СКЛЯНИНА}

Для треугольных и квазитреугольных супербиалгебр Ли можно получить соответствующие супергруппы Пуассона-Ли с помощью суперскобки Склянина, определяемой заданной суперкососимметричной $r$-матрицей $r=r^{i j} X_{i} \otimes X_{j}[6],[18]$

$$
\begin{aligned}
& \{f, h\}=f{\frac{\overleftarrow{\partial}}{\partial x^{\mu}}}^{\mu} X_{i}^{(\mathrm{L}, \mathrm{r})} r^{i j}{ }_{j} X^{(\mathrm{L}, 1)^{\nu}} \frac{\vec{\partial}}{\partial x^{\nu}} h-f \frac{\overleftarrow{\partial}}{\partial x^{\mu}}{ }^{\mu} X_{i}^{(\mathrm{R}, \mathrm{r})} r^{i j}{ }_{j} X^{(\mathrm{R}, \mathrm{l},)^{\nu}} \frac{\vec{\partial}}{\partial x^{\nu}} h \\
& \forall f, h \in C^{\infty}(G),
\end{aligned}
$$

где ${ }_{j} X^{(\mathrm{L}, \mathrm{l})}\left(X_{i}^{(\mathrm{L}, \mathrm{r})}\right)$ и ${ }_{j} X^{(\mathrm{R}, \mathrm{l})}\left(X_{i}^{(\mathrm{R}, \mathrm{r})}\right)$ - лево- и право-инвариантные супервекторные поля, включающие левые (правые) дифференцирования на супергруппе Пуассона-Ли $G$. Если $r$ является решением КУЯБ, то следуюшие суперскобки также являются суперпуассоновыми структурами на супергруппе $G[6]$ :

$$
\begin{aligned}
\{f, h\}^{\mathrm{L}} & =f{\frac{\overleftarrow{\partial}}{\partial x^{\mu}}}^{\mu} X_{i}^{(\mathrm{L}, \mathrm{r})} r^{i j}{ }_{j} X^{(\mathrm{L}, 1)^{\nu}} \frac{\vec{\partial}}{\partial x^{\nu}} h \\
\{f, h\}^{\mathrm{R}} & =f{\frac{\overleftarrow{\partial}}{\partial x^{\mu}}}^{\mu} X_{i}^{(\mathrm{R}, \mathrm{r})} r^{i j}{ }_{j} X^{(\mathrm{R}, \mathrm{l})^{\nu}} \frac{\vec{\partial}}{\partial x^{\nu}} h .
\end{aligned}
$$

Для вычисления левого и правого инвариантных супервекторных полей на супергруппе $G$ достаточно определить левые и правые инвариантные 1-формы. Для $g \in G$ имеем [19]

$$
\begin{aligned}
& g^{-1} d g=L_{i}^{i} X=(-1)^{i} L_{\mu}^{(\mathrm{r}) i} \overleftarrow{d x^{\mu}} X_{i}=(-1)^{i} \overrightarrow{d x^{\mu}}{ }_{\mu} L^{(1) i} X_{i}, \\
& d g g^{-1}=R_{i}^{i} X=(-1)^{i} R^{(\mathrm{r}) i}{ }_{\nu} \overleftarrow{d x^{\nu}} X_{i}=(-1)^{i} \overrightarrow{d x^{\nu}}{ }_{\nu} R^{(1) i} X_{i},
\end{aligned}
$$

где $x^{\mu}$ - координаты на супергруппе. Теперь, используя формулы [13]

$$
\left\langle\overrightarrow{i e}, \overrightarrow{d x^{j}}\right\rangle=\left\langle\frac{\vec{\partial}}{\partial x^{i}}, \overrightarrow{d x^{j}}\right\rangle={ }_{i} \delta^{j}, \quad\left\langle\overleftarrow{d x^{i}}, \overleftarrow{e_{j}}\right\rangle=\left\langle\overleftarrow{d x^{i}}, \frac{\overleftarrow{\partial}}{\partial x^{j}}\right\rangle={ }^{i} \delta_{j}
$$

в соотношениях

$$
\left\langle{ }_{j} X^{(\mathrm{L}(\mathrm{R}), \mathrm{l})}, L(R)^{(1) i}\right\rangle={ }_{j} \delta^{i}, \quad\left\langle L(R)^{(\mathrm{r}) i}, X_{j}^{(\mathrm{L}(\mathrm{R}), \mathrm{r})}\right\rangle={ }^{i} \delta_{j},
$$

где

$$
{ }_{j} X^{(\mathrm{L}(\mathrm{R}), \mathrm{l})}:={ }_{j} X^{(\mathrm{L}(\mathrm{R}), \mathrm{l})^{\mu}} \frac{\vec{\partial}}{\partial x^{\mu}}, \quad X_{j}^{(\mathrm{L}(\mathrm{R}), \mathrm{r})}:={\frac{\overleftarrow{\partial}}{\partial x^{\nu}}}^{\nu} X_{j}^{(\mathrm{L}(\mathrm{R}), \mathrm{r})}
$$

получаем следующие результаты:

$$
{ }_{j} X^{(\mathrm{L}(\mathrm{R}), \mathrm{l})^{\nu}}={ }_{j} L(R)^{(1)^{-1 \nu}}, \quad{ }^{\eta} X_{j}^{(\mathrm{L}(\mathrm{R}), \mathrm{r})}={ }^{\eta} L(R)_{j}^{(\mathrm{r})-1} .
$$

Чтобы вычислить приведенные выше матрицы, будем предполагать, что двумерная супергруппа $G$ имеет следующую параметризацию:

$$
g=e^{x X_{1}} e^{\psi X_{2}} .
$$


Аналогично для трехмерных супергрупп (с двумя бозонными и одним фермионным генераторами) полагаем

$$
g=e^{x X_{1}} e^{y X_{2}} e^{\psi X_{3}},
$$

а для трехмерных супергрупп (с двумя фермионными и одним бозонным генераторами) используем параметризацию

$$
g=e^{x X_{1}} e^{\psi X_{2}} e^{\chi X_{3}} .
$$

Результаты собраны в табл. 8, 9 (в этих таблицах для сокращения формул мы применяем обозначения $\frac{\vec{\partial}}{\partial \bullet}=\overrightarrow{\partial_{\bullet}}$ и $\frac{\overleftarrow{\partial}}{\partial \bullet}=\overleftarrow{\partial_{\bullet}}$ ). Чтобы получить эти результаты для дуальных супергрупп Ли $\widetilde{G}$, нужно воспользоваться следующими соотношениями ${ }^{5)}$ :

$$
\begin{aligned}
& \tilde{g}^{-1} d \tilde{g}=\tilde{L}_{i} \tilde{X}^{i}=\tilde{L}_{i \mu}^{(\mathrm{r})} \overleftarrow{d \tilde{x}^{\mu}} \widetilde{X}^{i}=\overrightarrow{d \tilde{x}^{\mu}}{ }_{\mu} \tilde{L}_{i}^{(1)} \widetilde{X}^{i} \\
& d \tilde{g} \tilde{g}^{-1}=\widetilde{R}_{i} \widetilde{X}^{i}=\widetilde{R}_{i \nu}^{(\mathrm{r})} \overleftarrow{d \tilde{x}^{\nu}} \widetilde{X}^{i}=\overrightarrow{d \tilde{x}^{\nu}}{ }_{\nu} \widetilde{R}_{i}^{(1)} \widetilde{X}^{i} \\
& \left\langle{ }^{j} \widetilde{X}^{(\mathrm{L}(\mathrm{R}), \mathrm{l})}, \tilde{L}_{i}\left(\widetilde{R}_{i}\right)^{(\mathrm{l})}\right\rangle={ }^{j} \delta_{i}, \quad\left\langle\tilde{L}_{i}\left(\widetilde{R}_{i}\right)^{(\mathrm{r})}, \widetilde{X}^{j(\mathrm{~L}(\mathrm{R}), \mathrm{r})}\right\rangle=\delta_{i}{ }^{j}, \\
& { }^{j} \widetilde{X}^{(\mathrm{L}(\mathrm{R}), \mathrm{l})}:={ }^{j} \widetilde{X}^{(\mathrm{L}(\mathrm{R}), \mathrm{l}) \mu} \frac{\vec{\partial}}{\partial \tilde{x}^{\mu}}, \quad \widetilde{X}^{j(\mathrm{~L}(\mathrm{R}), \mathrm{r})}:={\frac{\overleftarrow{\partial}}{\partial \tilde{x}^{\nu}}}^{\nu} \widetilde{X}^{j(\mathrm{~L}(\mathrm{R}), \mathrm{r})}, \\
& { }^{j} \widetilde{X}^{\mu(\mathrm{L}(\mathrm{R}), \mathrm{l})}={ }^{j} \tilde{L}(\widetilde{R})^{(\mathrm{l})^{-1} \mu}, \quad{ }^{\mu} X^{j(\mathrm{~L}(\mathrm{R}), \mathrm{r})}={ }^{\mu} \tilde{L}(\widetilde{R})^{(\mathrm{r})^{-1} j} .
\end{aligned}
$$

Заметим, что параметризация супергруппы Ли для $\widetilde{G}$ аналогична параметризации для $G$ (т. е. параметры $x, y, \psi, \chi$ заменяются на $\tilde{x}, \tilde{y}, \tilde{\psi}, \tilde{\chi})$.

Теперь можно вычислить суперпуассоновы структуры на супергруппах Ли $G$ и $\widetilde{G}$. Можно упростить соотношение (25), переписав его в матричном виде:

$$
\begin{aligned}
& \{f, g\}=\left(\begin{array}{lll}
f{\overleftarrow{X_{1}}}^{(\mathrm{L}, \mathrm{r})} & f{\overleftarrow{X_{2}}}^{(\mathrm{L}, \mathrm{r})} \quad f \overleftarrow{X_{3}}(\mathrm{~L}, \mathrm{r})
\end{array}\right) r\left(\begin{array}{l}
\overrightarrow{1}^{\vec{X}^{(\mathrm{L}, \mathrm{l})} g} g \\
\overrightarrow{2}^{(\mathrm{L}, \mathrm{l})} g \\
\overrightarrow{3}^{(\mathrm{L}, \mathrm{l})} g
\end{array}\right)- \\
& -\left(f{\overleftarrow{X_{1}}}^{(\mathrm{R}, \mathrm{r})} \quad f{\overleftarrow{X_{2}}}^{(\mathrm{R}, \mathrm{r})} f{\overleftarrow{X_{3}}}^{(\mathrm{R}, \mathrm{r})}\right) r\left(\begin{array}{l}
\overrightarrow{1}_{{ }^{(}{ }^{(\mathrm{R}, \mathrm{l})} g}^{\vec{X}^{(\mathrm{R}, \mathrm{l})} g} \\
\frac{{ }_{3} X^{(\mathrm{R}, l)} g}{}
\end{array}\right)
\end{aligned}
$$

и аналогично переписать $(26)$ и $(27)^{6)}$. Таким образом, мы вычисляем фундаментальную суперскобоку Пуассона для всех треугольных и квазитреугольных супербиалгебр Ли. Результаты приведены в табл. 10-13. Заметим, что для треугольных супербиалгебр Ли мы вычислили все суперпуассоновы структуры (25)-(27) и привели их в отдельных таблицах.

\footnotetext{
5) Причина, по которой мы не можем воспользоваться формулами (28)-(33) для дуальной супергруппы Ли $\widetilde{G}$, заключается в том, что для дуальных супералгебр Ли мы используем базис с верхними индексами и в обозначениях де Витта они отличаются от нижних индексов для супералгебр Ли.

6)Заметим, что для дуальной супергруппы Ли $\widetilde{G}$ мы используем суперскобку Склянина

$$
\{\tilde{f}, \tilde{h}\}=(-1)^{i}\left(\tilde{f} \frac{\overleftarrow{\partial}}{\partial \tilde{x}^{\mu}}{ }^{\mu} \tilde{X}^{i(\mathrm{~L}, \mathrm{r})} \tilde{r}_{i j}{ }^{j} \tilde{X}^{(L, l) \nu} \frac{\vec{\partial}}{\partial \tilde{x}^{\nu}} \tilde{h}-\tilde{f}{\frac{\overleftarrow{\partial}}{\partial \tilde{x}^{\mu}}}^{\mu} \tilde{X}^{i(\mathrm{R}, \mathrm{r})} \tilde{r}_{i j}{ }^{j} \tilde{X}^{(R, l) \nu} \frac{\vec{\partial}}{\partial \tilde{x}^{\nu}} \tilde{h}\right) \quad \forall \tilde{f}, \tilde{h} \in C^{\infty}(\widetilde{G})
$$
}


ТАБлица 8. Левые и правые инвариантные супервекторные поля на двумерных супергруппах Ли.

\begin{tabular}{|c|c|c|c|c|}
\hline $\mathrm{g}$ & $B$ & $\tilde{\mathrm{g}}$ & $\left(A_{1,1}+A\right)$ & $\left(A_{1,1}+A\right) \cdot i$ \\
\hline$\left({ }_{1} X^{(\mathrm{L}, \mathrm{l})}\right)$ & $\left(\overrightarrow{\partial_{x}}-\psi \overrightarrow{\partial_{\psi}}\right)$ & $\left({ }^{1} \widetilde{X}^{(\mathrm{L}, \mathrm{l})}\right)$ & $\overrightarrow{\partial_{\tilde{x}}}$ & $\overrightarrow{\partial_{\tilde{x}}}$ \\
\hline${ }_{2} X^{(\mathrm{L}, \mathrm{l})}$ & $-\overrightarrow{\partial_{\psi}}$ & ${ }^{2} \widetilde{X}^{(\mathrm{L}, \mathrm{l})}$ & & 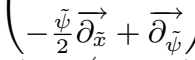 \\
\hline$\left(X_{1}^{(\mathrm{L}, \mathrm{r})}\right)$ & $\left(\overleftarrow{\partial_{x}}-\overleftarrow{\partial_{\psi}} \psi\right)$ & $\left(\widetilde{X}^{1(\mathrm{~L}, \mathrm{r})}\right)$ & $\overleftarrow{\partial_{\tilde{x}}}$ & $\overleftarrow{\partial_{\tilde{x}}}$ \\
\hline$X_{2}{ }^{(\mathrm{L}, \mathrm{r})}$ & $-\overleftarrow{\partial_{\psi}}$ & $\widetilde{X}^{2(\mathrm{~L}, \mathrm{r})}$ & $\overleftarrow{5}$ & $\frac{\tilde{\psi}}{2}-$ \\
\hline$\left({ }_{1} X^{(\mathrm{R}, 1)}\right)$ & $\int \overrightarrow{\partial_{x}}$ & $\left({ }^{1} \widetilde{X}^{(\mathrm{R}, 1)}\right)$ & $\overrightarrow{\partial_{\tilde{x}}}$ & $\overrightarrow{\partial_{\tilde{x}}}$ \\
\hline$\left({ }_{2} X^{(\mathrm{R}, \mathrm{l})}\right)$ & $-e^{-x} \overrightarrow{\partial_{\psi}}$ & ${ }^{2} \widetilde{X}^{(\mathrm{R}, 1)}$ & & $\frac{\tilde{\psi}}{2} \overrightarrow{\partial_{\tilde{x}}}+\vec{\partial}$ \\
\hline$\left(X_{1}^{(\mathrm{R}, \mathrm{r})}\right)$ & $\overleftarrow{\partial_{x}}$ & $\left(\tilde{X}^{1(\mathrm{R}, \mathrm{r})}\right)$ & $\overleftarrow{\partial_{\tilde{x}}}$ & $\overleftarrow{\partial_{\tilde{x}}}$ \\
\hline$\left(X_{2}^{(\mathrm{R}, \mathrm{r})}\right)$ & $\left(-\overleftarrow{\partial_{\psi}} e^{-x}\right)$ & $\left(\tilde{X}^{2(\mathrm{R}, \mathrm{r})}\right)$ & $-\overleftarrow{\partial_{\tilde{x}}} \frac{\tilde{\psi}}{2}-\overleftarrow{\partial_{\tilde{\psi}}}$ & $\left(\overleftarrow{\partial_{\tilde{x}}} \frac{\tilde{\psi}}{2}-\overleftarrow{\partial_{\tilde{\psi}}}\right)$ \\
\hline
\end{tabular}

ТАБлицА 9. Левые и правые инвариантные супервекторные поля на трехмерных супергруппах Ли.

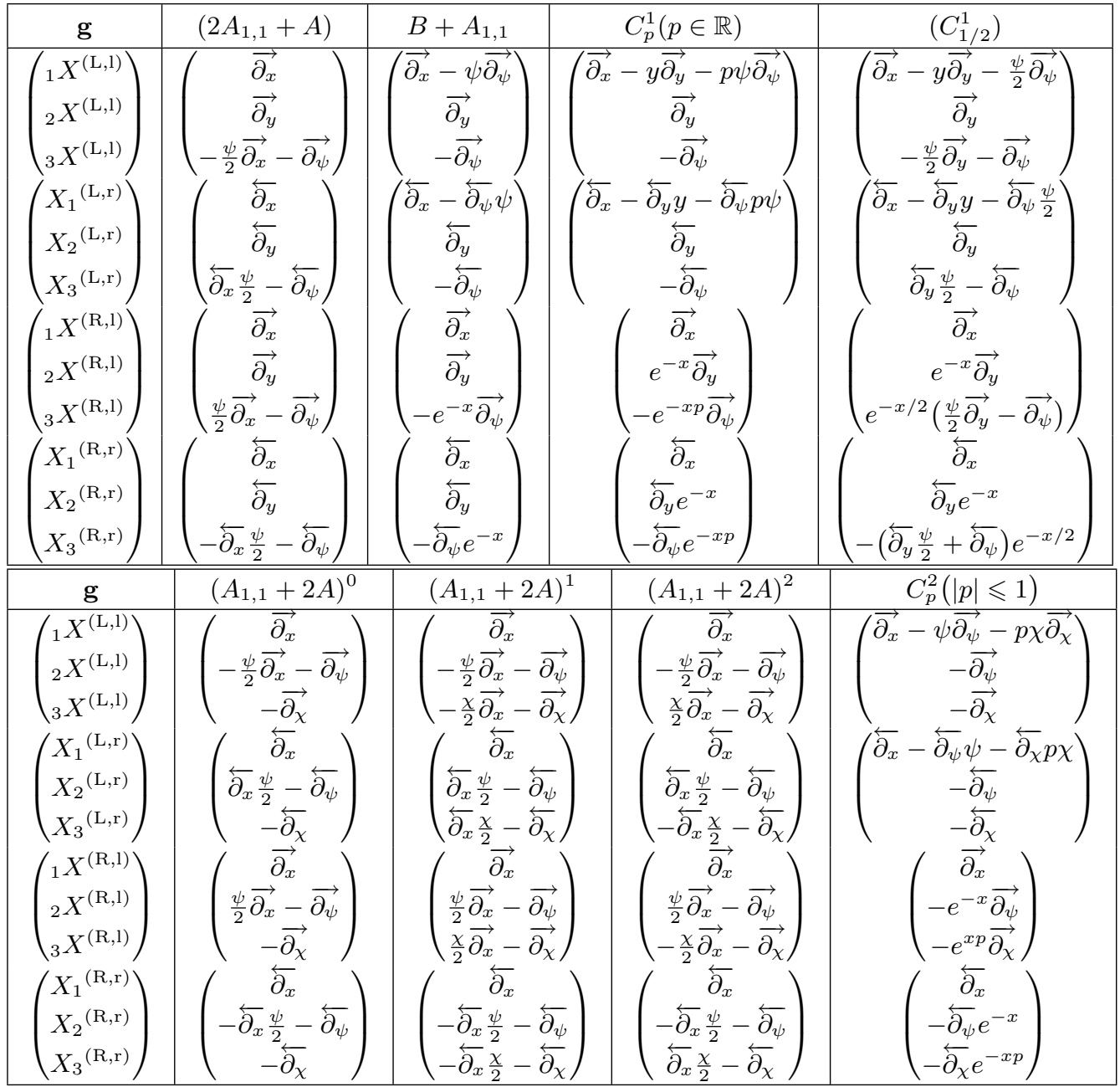


Продолжение табл. 9

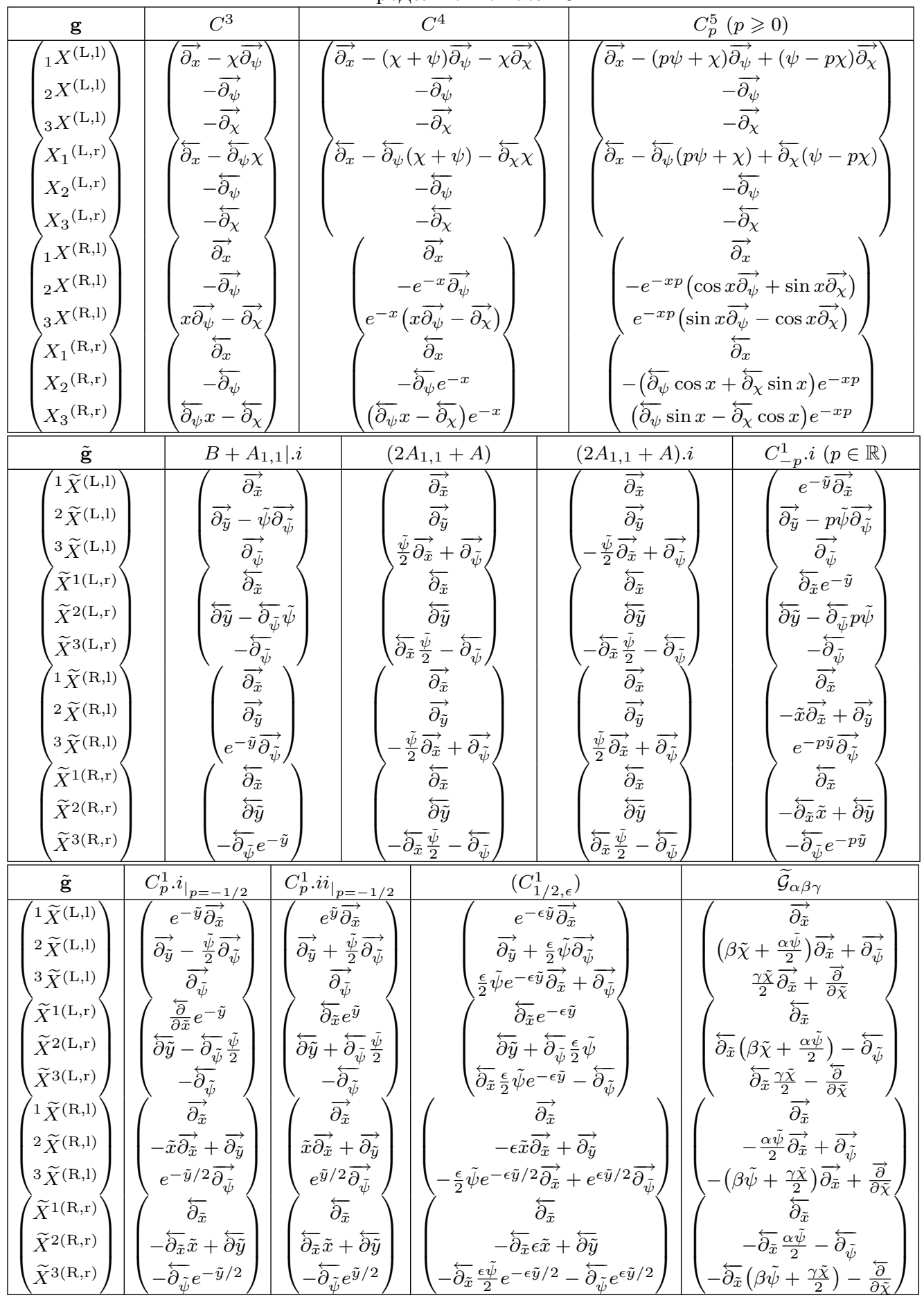


ТАБлицА 10. Суперскобки Пуассона, связанные с би- $r$-матричными, треугольными и квазитреугольными трехмерными супербиалгебрами Ли типа $(2,1)$.

\begin{tabular}{|c|c|c|c|c|c|c|}
\hline$(\mathrm{g}, \tilde{\mathbf{g}})$ & $\begin{array}{c}\left(C_{p}^{1}, C_{-p}^{1} . i\right) \\
\quad p=0\end{array}$ & $\begin{array}{l}\left(C_{p}^{1}, C_{-p}^{1} . i\right) \\
\quad p=1\end{array}$ & $\begin{array}{c}\left(C_{p}^{1}, C_{-p}^{1} . i\right) \\
\quad p=-1\end{array}$ & $\begin{array}{c}\left(C_{p}^{1}, C_{-p}^{1} . i\right) \\
\quad p \in \mathbb{R} \\
-\{0,1,-1\}\end{array}$ & $\begin{array}{l}\left(\left(C_{1 / 2}^{1}\right)\right. \\
\left.\left(C_{1 / 2, \epsilon}^{1}\right)\right)\end{array}$ & $\begin{array}{l}\left(B+A_{1,1}\right. \\
\left.B+A_{1,1} \mid \cdot i\right)\end{array}$ \\
\hline$\{x, y\}^{\mathrm{L}}$ & 1 & 1 & 1 & 1 & $\epsilon$ & 1 \\
\hline$\{x, \psi\}^{\mathrm{L}}$ & 0 & 0 & 0 & 0 & 0 & 0 \\
\hline$\{y, \psi\}^{\mathrm{L}}$ & 0 & $\psi-\zeta$ & $-\psi$ & $p \psi$ & $\epsilon \psi$ & $\psi$ \\
\hline$\{\psi, \psi\}^{\mathrm{L}}$ & $a$ & 0 & 0 & 0 & $-\epsilon$ & 0 \\
\hline$\{x, y\}^{\mathrm{R}}$ & $e^{-x}$ & $e^{-x}$ & $e^{-x}$ & $e^{-x}$ & $\epsilon e^{-x}$ & 1 \\
\hline$\{x, \psi\}^{\mathrm{R}}$ & 0 & 0 & 0 & 0 & 0 & 0 \\
\hline$\{y, \psi\}^{\mathrm{R}}$ & 0 & $-\zeta e^{-2 x}$ & 0 & 0 & $-\epsilon \frac{\psi}{2} e^{-x}$ & 0 \\
\hline$\{\psi, \psi\}^{\mathrm{R}}$ & $a$ & 0 & 0 & 0 & $-\epsilon e^{-x}$ & 0 \\
\hline$\{x, y\}$ & $1-e^{-x}$ & $1-e^{-x}$ & $1-e^{-x}$ & $1-e^{-x}$ & $\epsilon\left(1-e^{-x}\right)$ & 0 \\
\hline$\{x, \psi\}$ & 0 & 0 & 0 & 0 & 0 & 0 \\
\hline$\{y, \psi\}$ & 0 & $\psi-\zeta\left(1-e^{-2 x}\right.$ & $-\psi$ & $p \psi$ & $\epsilon \psi\left(1+\frac{e^{-x}}{2}\right)$ & $\psi$ \\
\hline$\{\psi, \psi\}$ & 0 & 0 & 0 & 0 & $-\epsilon\left(1-e^{-x}\right)$ & 0 \\
\hline$\{\tilde{x}, \tilde{y}\}^{\mathrm{L}}$ & $-e^{-\tilde{y}}$ & $-e^{-\tilde{y}}$ & $-e^{-\tilde{y}}$ & $-e^{-\tilde{y}}$ & $-\frac{e^{-\epsilon \tilde{y}}}{\epsilon}$ & -1 \\
\hline$\{\tilde{x}, \tilde{\psi}\}^{\mathrm{L}}$ & 0 & $\tilde{\psi} e^{-\tilde{y}}$ & $(\zeta-\tilde{\psi}) e^{-\tilde{y}}$ & $p \tilde{\psi} e^{-\tilde{y}}$ & $-\tilde{\psi} e^{-\epsilon \tilde{y}}$ & $\tilde{\psi}$ \\
\hline$\{\tilde{y}, \tilde{\psi}\}^{\mathrm{L}}$ & 0 & 0 & 0 & 0 & 0 & 0 \\
\hline$\{\tilde{\psi}, \tilde{\psi}\}^{\mathrm{L}}$ & $b$ & 0 & 0 & 0 & $\frac{1}{\epsilon}$ & 0 \\
\hline$\{\tilde{x}, \tilde{y}\}^{\mathrm{R}}$ & -1 & -1 & -1 & -1 & $-\frac{1}{\epsilon}$ & -1 \\
\hline$\{\tilde{x}, \tilde{\psi}\}^{\mathrm{R}}$ & 0 & 0 & $\zeta e^{\tilde{y}}$ & 0 & $\frac{\tilde{\psi}^{\tau}}{2}$ & 0 \\
\hline$\{\tilde{y}, \tilde{\psi}\}^{\mathrm{R}}$ & 0 & 0 & 0 & 0 & 0 & 0 \\
\hline$\{\tilde{\psi}, \tilde{\psi}\}^{\mathrm{R}}$ & $b$ & 0 & 0 & 0 & $\frac{1}{\epsilon} e^{\epsilon \tilde{y}}$ & 0 \\
\hline$\{\tilde{x}, \tilde{y}\}$ & $1-e^{-\tilde{y}}$ & $1-e^{-\tilde{y}}$ & $1-e^{-\tilde{y}}$ & $1-e^{-\tilde{y}}$ & $\frac{1}{\epsilon}\left(1-e^{-\epsilon \tilde{y}}\right)$ & 0 \\
\hline$\{\tilde{x}, \tilde{\psi}\}$ & 0 & $\tilde{\psi} e^{-\tilde{y}}$ & $-\tilde{\psi} e^{-\tilde{y}}-2 \zeta \operatorname{sh} \tilde{y}$ & $p \tilde{\psi} e^{-\tilde{y}}$ & $-\tilde{\psi}\left(\frac{1}{2}+e^{-\epsilon \tilde{y}}\right)$ & $\tilde{\psi}$ \\
\hline$\{\tilde{y}, \tilde{\psi}\}$ & 0 & 0 & 0 & 0 & 0 & 0 \\
\hline$\{\tilde{\psi}, \tilde{\psi}\}$ & 0 & 0 & 0 & 0 & $\frac{1}{\epsilon}\left(1-e^{\epsilon \tilde{y}}\right)$ & 0 \\
\hline$(\mathbf{g}, \tilde{\mathbf{g}})$ & \multicolumn{2}{|c|}{$\left(\left(C_{1 / 2}^{1}\right), C_{p=-1 / 2}^{1} \cdot i\right)$} & $\left(\left(C_{1 / 2}^{1}\right), C_{p=-1 / 2}^{1} . i i\right)$ & \multicolumn{2}{|c|}{$\begin{array}{c}\left(B+A_{1,1}\right. \\
\left.\left(2 A_{1,1}+A\right)\right)\end{array}$} & $\begin{array}{c}\left(B+A_{1,1},\right. \\
\left.\left(2 A_{1,1}+A\right) \cdot i\right)\end{array}$ \\
\hline$\{x, y\}^{\mathrm{L}}$ & \multicolumn{2}{|r|}{1} & -1 & \multicolumn{2}{|r|}{0} & 0 \\
\hline$\{x, \psi\}^{\mathrm{L}}$ & \multicolumn{2}{|r|}{0} & 0 & \multicolumn{2}{|r|}{0} & 0 \\
\hline$\{y, \psi\}^{\mathrm{L}}$ & \multicolumn{2}{|r|}{$\frac{\psi}{2}$} & $-\frac{\psi}{2}$ & \multicolumn{2}{|r|}{0} & 0 \\
\hline$\{\psi, \psi\}^{\mathrm{L}}$ & \multicolumn{2}{|r|}{$\begin{array}{l}2 \\
0\end{array}$} & $0^{2}$ & \multicolumn{2}{|r|}{$-\frac{1}{2}$} & $\frac{1}{2}$ \\
\hline$\{x, y\}^{\mathrm{R}}$ & \multicolumn{2}{|r|}{$e^{-x}$} & $-e^{-x}$ & \multicolumn{2}{|r|}{$0^{2}$} & 0 \\
\hline$\{x, \psi\}^{\mathrm{R}}$ & \multicolumn{2}{|r|}{0} & 0 & \multicolumn{2}{|r|}{0} & 0 \\
\hline$\{y, \psi\}^{\mathrm{R}}$ & \multicolumn{2}{|r|}{0} & 0 & \multicolumn{2}{|r|}{0} & 0 \\
\hline$\{\psi, \psi\}^{\mathrm{R}}$ & \multicolumn{2}{|r|}{0} & 0 & \multicolumn{2}{|c|}{$-\frac{1}{2} e^{-2 x}$} & $\frac{1}{2} e^{-2 x}$ \\
\hline$\{x, y\}$ & \multicolumn{2}{|c|}{$1-e^{-x}$} & $e^{-x}-1$ & \multicolumn{2}{|c|}{0} & 0 \\
\hline$\{x, \psi\}$ & \multicolumn{2}{|r|}{0} & 0 & \multicolumn{2}{|r|}{0} & 0 \\
\hline$\{y, \psi\}$ & \multicolumn{2}{|r|}{$\frac{\psi}{2}$} & $-\frac{\psi}{2}$ & & 0 & 0 \\
\hline$\{\psi, \psi\}$ & & 0 & $0^{2}$ & $-\frac{1}{2}(1$ & $\left.-e^{-2 x}\right)$ & $\frac{1}{2}\left(1-e^{-2 x}\right)$ \\
\hline$\{\tilde{x}, \tilde{y}\}$ & & $-e^{-\tilde{y}}$ & $e^{\tilde{y}}-1$ & & 0 & 0 \\
\hline$\{\tilde{x}, \tilde{\psi}\}$ & & $\frac{\tilde{\psi}}{2} e^{-\tilde{y}}$ & $\frac{\tilde{\psi}}{2} e^{\tilde{y}}$ & & $-\tilde{\psi}$ & $-\tilde{\psi}$ \\
\hline$\{\tilde{y}, \tilde{\psi}\}$ & & 0 & 0 & & 0 & 0 \\
\hline$\{\tilde{\psi}, \tilde{\psi}\}$ & & $-\tilde{y}-1$ & $1-e^{\tilde{y}}$ & & 0 & 0 \\
\hline
\end{tabular}


ТАБлица 11. Суперскобки Пуассона, связанные с треугольными и квазитреугольными трехмерными супербиалгебрами Ли типа $(2,1)$.

\begin{tabular}{|c|c|c|c|c|}
\hline$(\mathbf{g}, \tilde{\mathbf{g}})$ & $\left(\left(2 A_{1,1}+A\right), I_{(2,1)}\right)$ & $\left(C_{p=0}^{1}, I_{(2,1)}\right)$ & $\left(C_{p=1}^{1}, I_{(2,1)}\right)$ & $\left(C_{p=1}^{1},\left(2 A_{1,1}+A\right)\right)$ \\
\hline$\{x, y\}^{\mathrm{L}}$ & $b$ & 0 & 0 & 0 \\
\hline$\{x, \psi\}^{\mathrm{L}}$ & 0 & 0 & 0 & 0 \\
\hline$\{y, \psi\}^{\mathrm{L}}$ & 0 & 0 & $-\zeta$ & $-\zeta$ \\
\hline$\{\psi, \psi\}^{\mathrm{L}}$ & 0 & $a$ & 0 & $-\frac{1}{2}$ \\
\hline$\{x, y\}^{\mathrm{R}}$ & $b$ & 0 & 0 & 0 \\
\hline$\{x, \psi\}^{\mathrm{R}}$ & 0 & 0 & 0 & 0 \\
\hline$\{y, \psi\}^{\mathrm{R}}$ & 0 & 0 & $-\zeta e^{-2 x}$ & $-\zeta e^{-2 x}$ \\
\hline$\{\psi, \psi\}^{\mathrm{R}}$ & 0 & $a$ & 0 & $-\frac{1}{2} e^{-2 x}$ \\
\hline$\{x, y\}$ & 0 & 0 & 0 & 0 \\
\hline$\{x, \psi\}$ & 0 & 0 & 0 & 0 \\
\hline$\{y, \psi\}$ & 0 & 0 & $-\zeta\left(1-e^{-2 x}\right)$ & $-\zeta\left(1-e^{-2 x}\right)$ \\
\hline$\{\psi, \psi\}$ & 0 & 0 & 0 & $-\frac{1}{2}\left(1-e^{-2 x}\right)$ \\
\hline$(\mathbf{g}, \tilde{\mathbf{g}})$ & $\left(C_{p=1}^{1},\left(2 A_{1,1}+A\right) \cdot i\right)$ & $\left(C_{p}^{1},\left(2 A_{1,1}+A\right)\right)_{\left.\right|_{p \in \mathbb{R}-\{0,1\}}}$ & \multicolumn{2}{|c|}{$\left(C_{p}^{1},\left(2 A_{1,1}+A\right) \cdot i\right)_{\left.\right|_{p \in \mathbb{R}-\{0,1\}}}$} \\
\hline$\{x, y\}^{\mathrm{L}}$ & 0 & 0 & & 0 \\
\hline$\{x, \psi\}^{\mathrm{L}}$ & 0 & 0 & & 0 \\
\hline$\{y, \psi\}^{\mathrm{L}}$ & $-\zeta$ & 0 & & 0 \\
\hline$\{\psi, \psi\}^{\mathrm{L}}$ & $\frac{1}{2}$ & $-\frac{1}{2 p}$ & & $\frac{1}{2 p}$ \\
\hline$\{x, y\}^{\mathrm{R}}$ & 0 & 0 & & 0 \\
\hline$\{x, \psi\}^{\mathrm{R}}$ & 0 & 0 & & 0 \\
\hline$\{y, \psi\}^{\mathrm{R}}$ & $-\zeta e^{-2 x}$ & 0 & & 0 \\
\hline$\{\psi, \psi\}^{\mathrm{R}}$ & $\frac{1}{2} e^{-2 x}$ & $-\frac{1}{2 p} e^{-2 p x}$ & & $\frac{1}{2 p} e^{-2 p x}$ \\
\hline$\{x, y\}$ & 0 & 0 & & 0 \\
\hline$\{x, \psi\}$ & 0 & 0 & & 0 \\
\hline$\{y, \psi\}$ & $-\zeta\left(1-e^{-2 x}\right)$ & 0 & & 0 \\
\hline$\{\psi, \psi\}$ & $\frac{1}{2}\left(1-e^{-2 x}\right)$ & $-\frac{1}{2 p}\left(1-e^{-2 p x}\right)$ & & $\left(1-e^{-2 p x}\right)$ \\
\hline
\end{tabular}

ТАБлицА 12. Суперскобки Пуассона, связанные с треугольными и квазитреугольными двумерными супербиалгебрами Ли типа $(1,1)$

\begin{tabular}{|c|c|c|}
\hline$(\mathbf{g}, \tilde{\mathbf{g}})$ & $\left(B,\left(A_{1,1}+A\right)\right)$ & $\left(B,\left(A_{1,1}+A\right) \cdot i\right)$ \\
\hline$\{x, \psi\}^{\mathrm{L}}$ & 0 & 0 \\
$\{\psi, \psi\}^{\mathrm{L}}$ & $-\frac{1}{2}$ & $\frac{1}{2}$ \\
$\{x, \psi\}^{\mathrm{R}}$ & 0 & 0 \\
$\{\psi, \psi\}^{\mathrm{R}}$ & $-\frac{1}{2} e^{-2 x}$ & $\frac{1}{2} e^{-2 x}$ \\
$\{x, \psi\}$ & 0 & 0 \\
$\{\psi, \psi\}$ & $-\frac{1}{2}\left(1-e^{-2 x}\right)$ & $\frac{1}{2}\left(1-e^{-2 x}\right)$ \\
$\{\tilde{x}, \tilde{\psi}\}$ & $-\tilde{\psi}$ & $-\tilde{\psi}$ \\
$\{\tilde{\psi}, \tilde{\psi}\}$ & 0 & 0 \\
\hline
\end{tabular}


ТАБлицА 13. Суперскобки Пуассона, связанные с би- $r$-матричными треугольными трехмерными супербиалгебрами Ли типа $(1,2)$ (суперскобка Пуассона существует, если удовлетворяются условия, приведенные в графе "Комментарии").

\begin{tabular}{|c|c|c|c|c|c|c|c|}
\hline$(\mathbf{g}, \tilde{\mathbf{g}})$ & $\left(C_{p=0}^{2}, \widetilde{\mathcal{G}}_{\alpha, \beta, 0}\right)$ & $\begin{array}{c}\text { Ком- } \\
\text { мен- } \\
\text { тарии }\end{array}$ & \multicolumn{2}{|c|}{$\left(C_{p=-1}^{2}, \widetilde{\mathcal{G}}_{\alpha, 0, \gamma}\right)$} & $\begin{array}{c}\text { Ком- } \\
\text { мен- } \\
\text { тарии }\end{array}$ & $\left.\left(C_{p}^{2}, \widetilde{\mathcal{G}}_{\alpha \beta \gamma}\right)\right|_{p \in(-1,1]-\{0\}}$ & $\begin{array}{c}\text { Ком- } \\
\text { мен- } \\
\text { тарии }\end{array}$ \\
\hline$\{x, \psi\}^{\mathrm{L}}$ & 0 & & \multicolumn{2}{|l|}{0} & & 0 & \\
\hline$\{x, \chi\}^{\mathrm{L}}$ & 0 & & \multicolumn{2}{|l|}{0} & & 0 & \\
\hline$\{\psi, \chi\}^{\mathrm{L}}$ & $-\beta$ & & \multicolumn{2}{|l|}{$b$} & & $-\frac{\beta}{p+1}$ & \\
\hline$\{\psi, \psi\}^{\mathrm{L}}$ & $-\frac{\alpha}{2}$ & & \multicolumn{2}{|l|}{$-\frac{\alpha}{2}$} & & $-\frac{\alpha}{2}$ & \\
\hline$\{\chi, \chi\}^{\mathrm{L}}$ & $b$ & & \multicolumn{2}{|l|}{$\frac{\gamma}{2}$} & & $-\frac{\gamma}{2 p}$ & \\
\hline$\{x, \psi\}^{\mathrm{R}}$ & \multirow{2}{*}{$\begin{array}{l}0 \\
0\end{array}$} & & \multicolumn{2}{|l|}{0} & & 0 & \\
\hline$\{x, \chi\}^{\mathrm{R}}$ & & & \multicolumn{2}{|l|}{0} & & 0 & \\
\hline$\{\psi, \chi\}^{\mathrm{R}}$ & $-\beta e^{-x}$ & & \multicolumn{2}{|c|}{$\begin{array}{c}b \\
\alpha-2 x\end{array}$} & & $-\frac{\beta}{p+1} e^{-x(1+p)}$ & \\
\hline$\{\psi, \psi\}^{\mathrm{R}}$ & \multirow{2}{*}{$-\frac{\alpha}{2} e^{-2 x}$} & & \multirow{2}{*}{\multicolumn{2}{|c|}{$\begin{array}{l}-\frac{\alpha}{2} e^{-2 x} \\
\frac{\gamma}{\gamma} e^{2 x}\end{array}$}} & & $-\frac{\alpha}{2} e^{-2 x}$ & \\
\hline$\{\chi, \chi\}^{\mathrm{R}}$ & & & & & & $-\frac{\gamma}{2 p} e^{-2 x p}$ & \\
\hline$\{x, \psi\}$ & 0 & & \multicolumn{2}{|c|}{$\begin{array}{r}2 \\
0\end{array}$} & & 0 & \\
\hline$\{x, \chi\}$ & \multirow{2}{*}{$\begin{array}{c}0 \\
\beta\left(e^{-x}-1\right)\end{array}$} & & \multicolumn{2}{|c|}{0} & & 0 & \\
\hline$\{\psi, \chi\}$ & & & 0 & & & $\frac{\beta}{p+1}\left(e^{-x(1+p)}-1\right)$ & \\
\hline$\{\psi, \psi\}$ & $\begin{array}{l}\beta\left(e^{-x}-1\right) \\
\underline{\alpha}\left(e^{-2 x}-1\right)\end{array}$ & & $\frac{\alpha}{2}\left(e^{-2 x}\right.$ & & & $\frac{\alpha}{2}\left(e^{-2 x}-1\right)$ & \\
\hline$\{\chi, \chi\}$ & 0 & & $\frac{\gamma}{2}(1-e$ & & & $\frac{\gamma}{2 p}\left(e^{-2 x p}-1\right)$ & \\
\hline$\{\tilde{x}, \tilde{\psi}\}$ & $-\tilde{\psi}$ & & $-\tilde{\psi}$ & & & $-\tilde{\psi}$ & \\
\hline$\{\tilde{x}, \tilde{\chi}\}$ & 0 & если & $\tilde{\chi}$ & & если & $-p \tilde{\chi}$ & если \\
\hline$\{\tilde{\psi}, \underset{\sim}{\tilde{\chi}}\}$ & 0 & $\beta=0$ & 0 & & $\alpha, \gamma \neq 0$ & 0 & $\beta=0$ \\
\hline$\{\tilde{\psi}, \tilde{\psi}\}$ & 0 & $\alpha \neq 0$ & 0 & & & 0 & $\alpha, \gamma \neq 0$ \\
\hline$\{\tilde{\chi}, \tilde{\chi}\}$ & 0 & & 0 & & & 0 & \\
\hline$(\mathbf{g}, \tilde{\mathbf{g}})$ & $\left(C^{3}, I_{(1,2)}\right)$ & $\left(C^{3},\left(A_{1,1}\right.\right.$ & $\left.+2 A)_{1,0,0}^{0}\right)$ & & & $\left.C^{4}, \widetilde{\mathcal{G}}_{\alpha \beta \gamma}\right)$ & $\begin{array}{c}\text { Ком- } \\
\text { мен- } \\
\text { тарии }\end{array}$ \\
\hline$\{x, \psi\}^{\mathrm{L}}$ & $-\zeta$ & & 5 & & & 0 & \\
\hline$\{x, \chi\}^{\mathrm{L}}$ & 0 & & 0 & & & 0 & \\
\hline$\{\psi, \chi\}^{\mathrm{L}}$ & 0 & & $\frac{1}{2}$ & & & $\frac{\gamma-2 \beta}{4}$ & \\
\hline$\{\psi, \psi\}^{\mathrm{L}}$ & $c$ & & $b$ & & & $\frac{2(\beta-\alpha)-\gamma}{4}$ & \\
\hline$\{\chi, \chi\}^{\mathrm{L}}$ & 0 & & 0 & & & $-\frac{\gamma}{2}$ & \\
\hline$\{x, \psi\}^{\mathrm{R}}$ & $-\zeta$ & & ) & & & 0 & \\
\hline$\{x, \chi\}^{\mathrm{R}}$ & 0 & & b & & & 0 & \\
\hline$\{\psi, \chi\}^{\mathrm{R}}$ & 0 & & $\frac{1}{2}$ & & $\frac{e^{-2 x}}{4}[2$ & $2 \gamma x+\gamma-2 \beta]$ & \\
\hline$\{\psi, \psi\}^{\mathrm{R}}$ & $c$ & & $-x$ & $\frac{e^{-2 x}}{2}$ & {$\left[-\gamma x^{2}+(\right.$} & $\left.(2 \beta-\gamma) x+\beta-\alpha-\frac{\gamma}{2}\right]$ & \\
\hline$\{\chi, \chi\}^{\mathrm{R}}$ & 0 & & 0 & & & $\frac{-\gamma}{2} e^{-2 x}$ & \\
\hline$\{x, \psi\}$ & 0 & & D & & & 0 & \\
\hline$\{x, \chi\}$ & 0 & & ) & & & 0 & \\
\hline$\{\psi, \chi\}$ & 0 & & ) & & $(\gamma-2 \beta)(1$ & $\left.\left.-e^{-2 x}\right)-2 \gamma x e^{-2 x}\right]$ & \\
\hline$\{\psi, \psi\}$ & 0 & & $x$ & & $\frac{1}{2}[(\beta-\alpha-$ & $\left.-\frac{\gamma}{2}\right)\left(1-e^{-2 x}\right)+$ & \\
\hline$\{\chi, \chi\}$ & 0 & & J & & $\frac{\gamma}{2}($ & $\left(e^{-2 x}-1\right)$ & \\
\hline$\{\tilde{x}, \tilde{\psi}\}$ & - & & 0 & & & $-\tilde{\psi}$ & \\
\hline$\{\underset{\sim}{\tilde{x}} \tilde{\chi}\}$ & - & & $\tilde{\psi}$ & & & $-(\tilde{\psi}+\tilde{\chi})$ & если \\
\hline$\{\tilde{\psi}, \tilde{\chi}\}$ & - & & 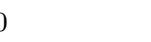 & & & 0 & $\beta \neq 0$ \\
\hline$\{\tilde{\psi}, \tilde{\psi}\}$ & - & & 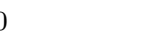 & & & 0 & $\alpha=\gamma=0$ \\
\hline$\{\tilde{\chi}, \tilde{\chi}\}$ & - & & b & & & 0 & \\
\hline
\end{tabular}


Продолжение табл. 13

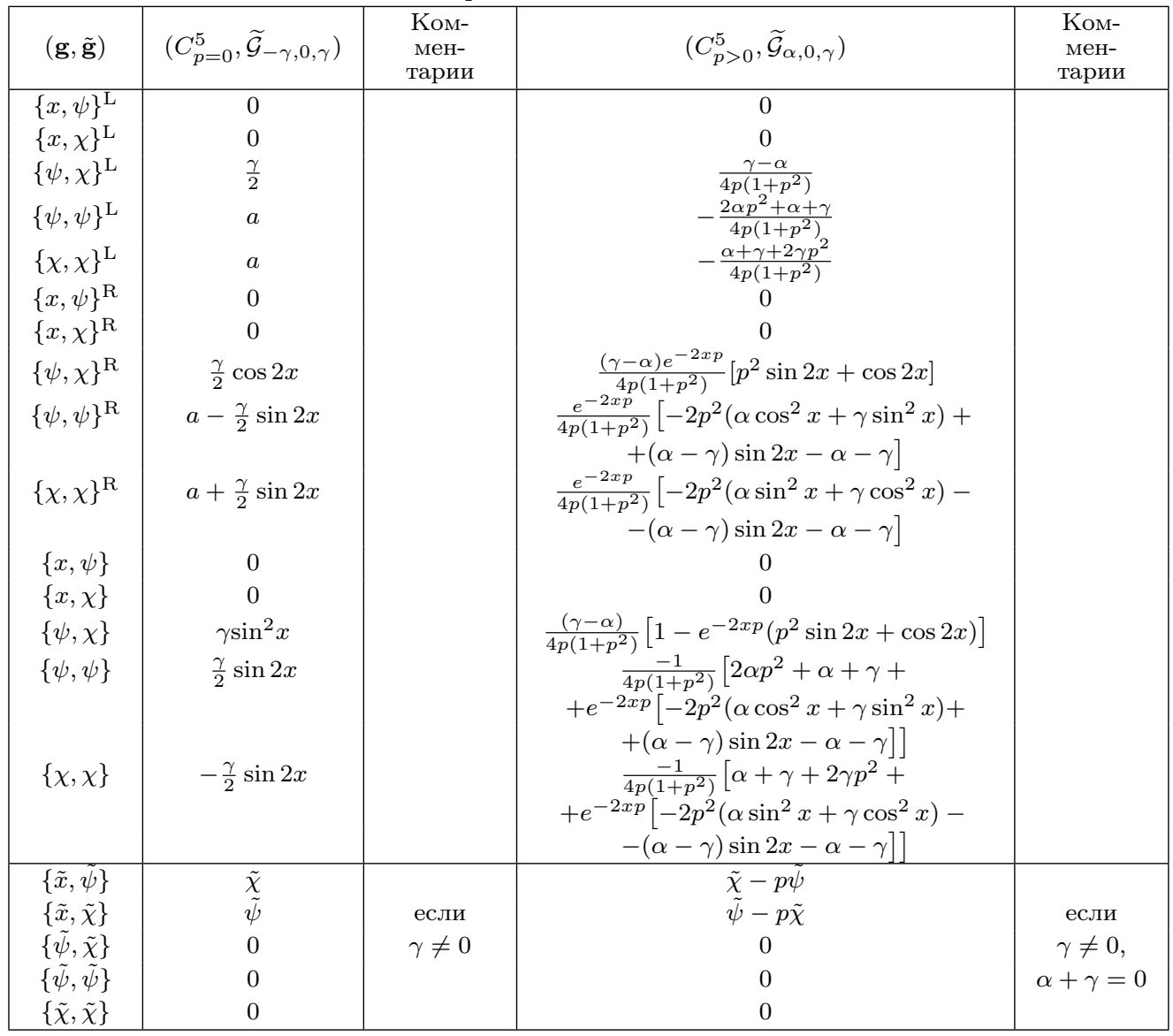

\begin{tabular}{|c|c|c|c|}
\hline$(\mathbf{g}, \tilde{\mathbf{g}})$ & $\left(C^{3},\left(A_{1,1}+2 A\right)_{0, \epsilon, 0}^{2}\right)$ & $(\mathbf{g}, \tilde{\mathbf{g}})$ & $\left(\left(A_{1,1}+2 A\right)^{0}, I_{(1,2)}\right)$ \\
\hline$\{x, \psi\}$ & 0 & $\{x, \psi\}^{\mathrm{L}}$ & 0 \\
$\{x, \chi\}$ & 0 & $\{x, \chi\}^{\mathrm{L}}$ & 0 \\
$\{\psi, \chi\}$ & $-\epsilon x$ & $\{\psi, \chi\}^{\mathrm{L}}$ & 0 \\
$\{\psi, \psi\}$ & $\epsilon x^{2}$ & $\{\psi, \psi\}^{\mathrm{L}}$ & $d, \chi\}^{\mathrm{L}}$ \\
$\{\chi, \chi\}$ & 0 & $\{x, \psi\}^{\mathrm{R}}$ & 0 \\
$\{\tilde{x}, \tilde{\psi}\}^{\mathrm{L}}$ & 0 & $\{x, \chi\}^{\mathrm{R}}$ & $-\zeta$ \\
$\{\tilde{x}, \tilde{\chi}\}^{\mathrm{L}}$ & 0 & $\{\psi, \chi\}^{\mathrm{R}}$ & 0 \\
$\{\tilde{\psi}, \tilde{\chi}\}^{\mathrm{L}}$ & 0 & $\{\psi, \psi\}^{\mathrm{R}}$ & 0 \\
$\{\tilde{\psi}, \tilde{\psi}\}^{\mathrm{L}}$ & 0 & $\{\chi, \chi\}^{\mathrm{R}}$ & 0 \\
$\{\tilde{\chi}, \tilde{\chi}\}^{\mathrm{L}}$ & $1 / \epsilon$ & $\{x, \psi\}$ & 0 \\
$\{\tilde{x}, \tilde{\psi}\}^{\mathrm{R}}$ & 0 & $\{x, \chi\}$ & 0 \\
$\{\tilde{x}, \tilde{\chi}\}^{\mathrm{R}}$ & $\tilde{\psi}$ & $\{\psi, \chi\}$ & 0 \\
$\{\tilde{\psi}, \tilde{\chi}\}^{\mathrm{R}}$ & 0 & $\{\psi, \psi\}$ & \\
$\{\tilde{\psi}, \tilde{\psi}\}^{\mathrm{R}}$ & 0 & $\{\chi, \chi\}$ & \\
$\{\tilde{\chi}, \tilde{\chi}\}^{\mathrm{R}}$ & $1 / \epsilon$ & & \\
$\{\tilde{x}, \tilde{\psi}\}$ & 0 & & \\
$\{\tilde{x}, \tilde{\chi}\}$ & $-\tilde{\psi}$ & & \\
$\{\tilde{\psi}, \tilde{\chi}\}$ & 0 & & \\
$\{\tilde{\psi}, \tilde{\psi}\}$ & 0 & & \\
$\{\tilde{\chi}, \tilde{\chi}\}$ & 0 & & \\
\hline
\end{tabular}




\section{6. ЗАКЛЮЧЕНИЕ}

Определив типы (треугольные, квазитреугольные или факторизуемые) двух- и трехмерных супербиалгебр Ли и получив их $r$-матрицы и суперпуассоновы структуры, мы сможем выполнить квантование данных супербиалгебр Ли. Более того, теперь мы можем исследовать интегрируемость в условиях супер-Т-дуальности Пуассона-Ли, изучая супер-Т-дуальные сигма-модели Пуассона-Ли [9] над би- $r$-матричными супербиалгебрами.

Благодарности. Работа выполнена при поддержке Research Vice Chancellor of Azarbaijan Shahid Madani University. Авторы выражают благодарность С. Могадасси за внимательное прочтение рукописи и полезные замечания.

\section{Список литературы}

[1] В. Г. Дринфельд, Докл. АН СССР, 268:2 (1983), 285-287.

[2] P. Etingof, D. Kazhdan, Selecta Math., 2:1 (1996), 1-41; 4:2 (1998), 213-231.

[3] Y. Kosmann-Schwarzbach, "Lie bialgebras, Poisson Lie groups and dressing transformations", Integrability of Nonlinear Systems, Proceedings of the CIMPA International School (Pondicherry, January 8-26, 1996), Lecture Notes in Physics, 495, eds. Y. Kosmann-Schwarzbach, B. Grammaticos, K. M. Tamizhmani, Springer, Berlin, 1996, 104-170.

[4] C. Klimčik, P. Ševera, Phys. Lett. B, 351:4 (1995), 455-462, arXiv: hep-th/9502122; Nucl. Phys. B Proc. Suppl., 46 (1996), 116-121, arXiv: hep-th/9509095.

[5] С. Е. Пархоменко, ЖЭТФ, 102:1 (1992), 3-7; E. Getzler, Manin triples and $N=2$ superconformal field theory, arXiv: hep-th/9307041.

[6] N. Andruskiewitsch, Abh. Math. Sem. Univ. Hamburg, 63 (1993), 147-163.

[7] N. Beisert, E. Spill, Commun. Math. Phys., 285:2 (2009), 537-565, arXiv: 0708.1762.

[8] J. M. Evans, J. O. Madsen, Nucl. Phys. B, 503:3 (1997), 715-746, arXiv: hep-th/9703065.

[9] A. Eghbali, A. Rezaei-Aghdam, JHEP, 09 (2009), 094, 14 pp., arXiv: 0901.1592.

[10] N. Geer, Etingof-Kazdan quantization of Lie superbialgebras, arXiv: math.QA/0409563.

[11] A. Eghbali, A. Rezaei-Aghdam, F. Heidarpour, J. Math. Phys., 51:7 (2010), 073503, 14 pp., arXiv: 0901.4471.

[12] A. Eghbali, A. Rezaei-Aghdam, F. Heidarpour, J. Math. Phys., 51:10 (2010), 103503, 33 pp., arXiv: 0911.1760.

[13] B. DeWitt, Supermanifolds, Cambridge Univ. Press, Cambridge, 1992.

[14] A. Rezaei-Aghdam, M. Hemmati, A. R. Rastkar, J. Phys. A, 38:18 (2005), 3981-3994, arXiv: math-ph/0412092.

[15] M. A. Jafarizadeh, A. Rezaei-Aghdam, Phys. Lett. B, 458:4 (1999), 477-490, arXiv: hep-th/9903152.

[16] L. Šnobl, L. Hlavatý, Int. J. Modern Phys. A, 17:28 (2002), 4043-4068, arXiv: math.QA/0202210.

[17] N. Backhouse, J. Math. Phys., 19:11 (1978), 2400-2402.

[18] C. Juszczak, J. T. Sobczyk, J. Math. Phys., 39:9 (1998), 4982-4992.

[19] P. Kosiński, J. Lukierski, P. Maślanka, J. Sobczyk, J. Phys. A, 27:20 (1994), 6827-6837, arXiv: hep-th/9405076.

Поступила в редакцию 18.06.2011, после доработки 13.09.2011 